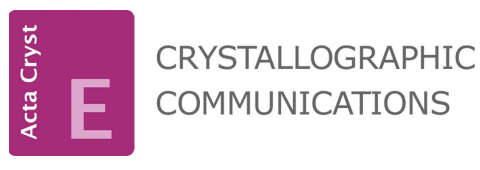

ISSN 2056-9890

\section{Crystal structures of three hydrogen-bonded 1:2 compounds of chloranilic acid with 2-pyridone, 3-hydroxypyridine and 4-hyroxypyridine}

\author{
Kazuma Gotoh and Hiroyuki Ishida*
}

Department of Chemistry, Faculty of Science, Okayama University, Okayama 700-8530, Japan. *Correspondence e-mail: ishidah@cc.okayama-u.ac.jp

Received 17 September 2017

Accepted 21 September 2017

Edited by A. J. Lough, University of Toronto, Canada

Keywords: crystal structure; chloranilic acid; 2-pyridone; 3-hydroxypyridine;

4-hyroxypyridine; hydrogen bond.

CCDC references: $1575722 ; 1575721$; 1575720

Supporting information: this article has supporting information at journals.iucr.org/e

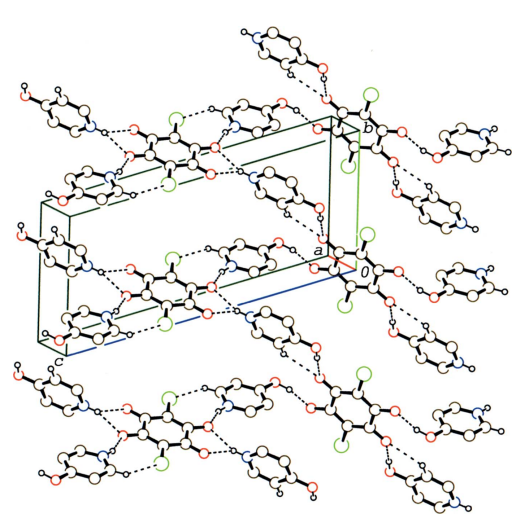

OPEN $\odot$ ACCESS
The crystal structures of the 1:2 compounds of chloranilic acid (systematic name: 2,5-dichloro-3,6-dihydroxy-1,4-benzoquinone) with 2-pyridone, 3-hydroxypyridine and 4-hyroxypyridine, namely, bis(2-pyridone) chloranilic acid, $2 \mathrm{C}_{5} \mathrm{H}_{5} \mathrm{NO} \cdot \mathrm{C}_{6} \mathrm{H}_{2} \mathrm{Cl}_{2} \mathrm{O}_{4}$, (I), bis(3-hydroxypyridinium) chloranilate, $2 \mathrm{C}_{5} \mathrm{H}_{6} \mathrm{NO}^{+}$.$\mathrm{C}_{6} \mathrm{Cl}_{2} \mathrm{O}_{4}{ }^{2-}$, (II), and bis(4-hydroxypyridinium) chloranilate, $2 \mathrm{C}_{5} \mathrm{H}_{6} \mathrm{NO}^{+}$.$\mathrm{C}_{6} \mathrm{Cl}_{2} \mathrm{O}_{4}{ }^{2-}$, (III), have been determined at $120 \mathrm{~K}$. In the crystal of (I), the base molecule is in the lactam form and no acid-base interaction involving $\mathrm{H}$-atom transfer is observed. The acid molecule lies on an inversion centre and the asymmetric unit consists of one half-molecule of chloranilic acid and one 2-pyridone molecule, which are linked via a short $\mathrm{O}-\mathrm{H} \cdots \mathrm{O}$ hydrogen bond. 2-Pyridone molecules form a head-to-head dimer via a pair of $\mathrm{N}-\mathrm{H} \cdots \mathrm{O}$ hydrogen bonds, resulting in a tape structure along [201]. In the crystals of (II) and (III), acid-base interactions involving $\mathrm{H}$-atom transfer are observed and the divalent cations lie on an inversion centre. The asymmetric unit of (II) consists of one half of a chloranilate anion and one 3-hydroxypyridinium cation, while that of (III) comprises two independent halves of anions and two 4-hydroxypyridinium cations. The primary intermolecular interaction in (II) is a bifurcated $\mathrm{O}-\mathrm{H} \cdots(\mathrm{O}, \mathrm{O})$ hydrogen bond between the cation and the anion. The hydrogen-bonded units are further linked via $\mathrm{N}-\mathrm{H} \cdots \mathrm{O}$ hydrogen bonds, forming a layer parallel to the $b c$ plane. In (III), one anion is surrounded by four cations via $\mathrm{O}-\mathrm{H} \cdots \mathrm{O}$ and $\mathrm{C}-\mathrm{H} \cdots \mathrm{O}$ hydrogen bonds, while the other is surrounded by four cations via $\mathrm{N}-\mathrm{H} \cdots \mathrm{O}$ and $\mathrm{C}-\mathrm{H} \cdots \mathrm{Cl}$ hydrogen bonds. These interactions link the cations and the anions into a layer parallel to (301).

\section{Chemical context}

Chloranilic acid, a dibasic acid with hydrogen-bond donor and acceptor groups, appears particularly attractive as a template for generating tightly bound self-assemblies with various pyridine derivatives, as well as a model compound for investigating hydrogen transfer motions in $\mathrm{O}-\mathrm{H} \cdots \mathrm{N}$ and $\mathrm{N}-$ H. . O hydrogen-bond systems (Zaman et al., 2004; Seliger et al., 2009; Asaji et al. 2010). In the present study, we have prepared three 1:2 compounds of chloranilic acid with 2-pyridone, 3-hydroxypyridine and 4-hydroxypyridine in order to extend our study of $D-\mathrm{H} \cdots A$ hydrogen bonding $(D$ $=\mathrm{N}, \mathrm{O}$ or $\mathrm{C} ; A=\mathrm{N}, \mathrm{O}$ or $\mathrm{Cl}$ ) in chloranilic acid-substitutedpyridine systems (Gotoh et al., 2009a,b, 2010). The crystal structure of the 1:1 compound of chloranilic acid with 3-hydroxypyridine, namely, 3-hydroxypyridinium hydrogen chloranilate monohydrate, has been reported (Gotoh \& Ishida, 2009). 
<smiles>O=c1cccc[nH]1</smiles><smiles>O=C1C(O)=C(Cl)C(=O)C(O)=C1Cl</smiles>

(I)<smiles>Oc1cccnc1</smiles>

(II)<smiles>O=C1C([O-])=C(Cl)C(=O)C([O-])=C1Cl</smiles><smiles>Oc1ccncc1</smiles><smiles>O=C1C([O-])=C(Cl)C(=O)C(Cl)=C1Cl</smiles>

(III)

\section{Structural commentary}

In compound (I), the base molecule is in the lactam form and no acid-base interaction involving $\mathrm{H}$-atom transfer is observed (Fig. 1). The acid molecule lies on an inversion centre and the asymmetric unit consists of one-half acid molecule and one base molecule, which are linked via a short $\mathrm{O}-\mathrm{H} \cdots \mathrm{O}$ hydrogen bond $(\mathrm{O} 2-\mathrm{H} 2 \cdots \mathrm{O} 3$; Table 1$)$. The dihedral angle between the acid ring and the base ring is $37.82(5)^{\circ}$.

In compound (II), an acid-base interaction involving $\mathrm{H}$-atom transfer is observed. The chloranilate anion is located on an inversion centre and the asymmetric unit contains onehalf anion molecule and one cation molecule. The primary intermolecular interaction between the cation and the anion is a bifurcated $\mathrm{O}-\mathrm{H} \cdots(\mathrm{O}, \mathrm{O})$ hydrogen bond $(\mathrm{O} 3-\mathrm{H} 3 \cdots \mathrm{O} 2$

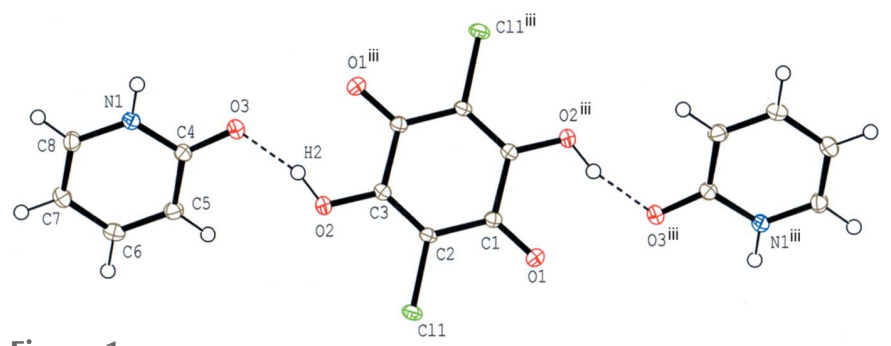

Figure 1

The molecular structure of compound (I), showing the atom-numbering scheme. Displacement ellipsoids are drawn at the $50 \%$ probability level and $\mathrm{H}$ atoms are shown as small spheres of arbitrary radii. $\mathrm{O}-\mathrm{H} \cdots \mathrm{O}$ hydrogen bonds are shown as dashed lines. [Symmetry code: (iii) $-x+1$, $-y+1,-z+1$.]
Table 1

Hydrogen-bond geometry $\left(\AA,^{\circ}\right)$ for (I).

\begin{tabular}{lllll}
\hline$D-\mathrm{H} \cdots A$ & $D-\mathrm{H}$ & $\mathrm{H} \cdots A$ & $D \cdots A$ & $D-\mathrm{H} \cdots A$ \\
\hline $\mathrm{O} 2-\mathrm{H} 2 \cdots \mathrm{O} 3$ & $0.901(15)$ & $1.627(16)$ & $2.4989(11)$ & $161.9(19)$ \\
$\mathrm{N}^{\mathrm{H}}-\mathrm{H} 1 \cdots \mathrm{O} 3^{\mathrm{i}}$ & $0.893(16)$ & $1.996(17)$ & $2.8743(12)$ & $167.6(16)$ \\
${\text { C7-H7 } \cdots \text { Cl1 }^{\text {ii }}}$ & 0.95 & 2.79 & $3.5122(13)$ & 134 \\
\hline
\end{tabular}

Symmetry codes: (i) $-x+2, y,-z+\frac{3}{2}$; (ii) $-x+2,-y,-z+1$.

and $\mathrm{O} 3-\mathrm{H} 3 \cdots \mathrm{O} 1^{\mathrm{i}}$; symmetry code as in Table 2) to afford a centrosymmetric 1:2 aggregate of the anion and the cation (Fig. 2). The dihedral angle between the acid ring and the base ring is $72.69(5)^{\circ}$.

The compound (III) crystallizes with two independent halves of chloranilate anions and two 4-hydroxypyridinium cations in the asymmetric unit (Fig. 3). Although both anions lie on an inversion centre, the hydrogen-bonding schemes around the anions are quite different (Fig. 4); one anion is surrounded by four cations via $\mathrm{O}-\mathrm{H} \cdots \mathrm{O}$ and $\mathrm{C}-\mathrm{H} \cdots \mathrm{O}$ hydrogen bonds $\left(\mathrm{O} 5-\mathrm{H} 5 \cdots \mathrm{O} 1^{\mathrm{i}}, \mathrm{O} 6-\mathrm{H} 6 \cdots \mathrm{O} 2\right.$ and $\mathrm{C} 13-$ $\mathrm{H} 13 . . \mathrm{O} 2$; symmetry code as in Table 3), while the other is surrounded by four cation via $\mathrm{N}-\mathrm{H} \cdots \mathrm{O}$ and $\mathrm{C}-\mathrm{H} \cdots \mathrm{Cl}$ hydrogen bonds $\left(\mathrm{N} 1-\mathrm{H} 1 \cdots \mathrm{O} 4, \mathrm{~N} 2-\mathrm{H} 2 \cdots \mathrm{O} 4^{\mathrm{ii}}, \quad \mathrm{N} 2-\right.$ $\mathrm{H} 2 \cdots \mathrm{O} 3{ }^{\text {iii }}$ and $\mathrm{C} 7-\mathrm{H} 7 \cdots \mathrm{Cl} 2$; Table 3$)$.

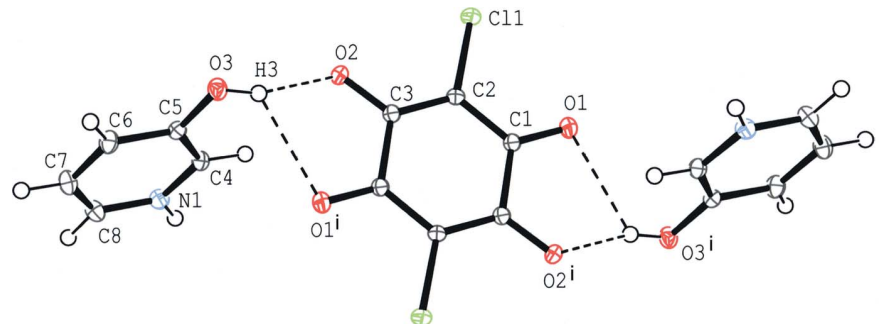

Figure 2

The molecular structure of compound (II), showing the atom-numbering scheme. Displacement ellipsoids are drawn at the $50 \%$ probability level and $\mathrm{H}$ atoms are shown as small spheres of arbitrary radii. $\mathrm{O}-\mathrm{H} \cdots \mathrm{O}$ hydrogen bonds are shown as dashed lines. [Symmetry code: (i) $-x+2$, $-y+1,-z+1$.]

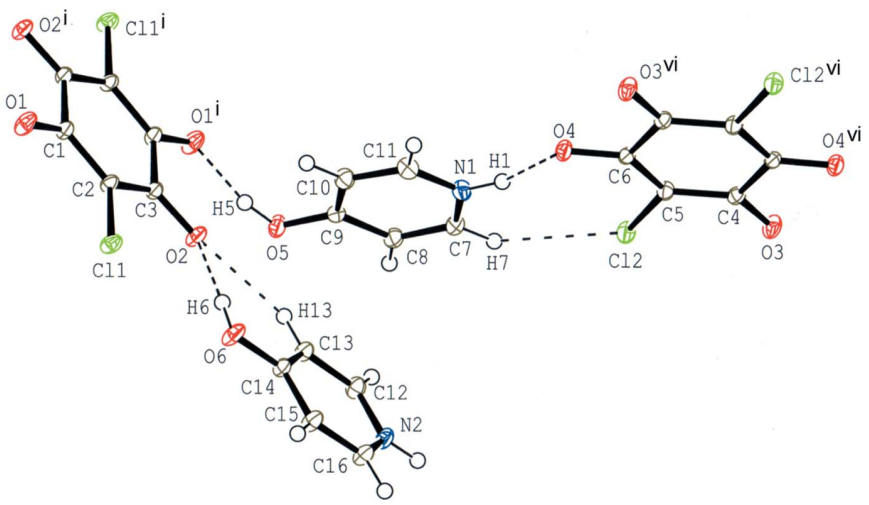

Figure 3

The molecular structure of compound (III), showing the atom-numbering scheme. Displacement ellipsoids are drawn at the $50 \%$ probability level and $\mathrm{H}$ atoms are shown as small spheres of arbitrary radii. $\mathrm{O}-\mathrm{H} \cdots \mathrm{O}$, $\mathrm{N}-\mathrm{H} \cdots \mathrm{O}, \mathrm{C}-\mathrm{H} \cdots \mathrm{Cl}$ and $\mathrm{C}-\mathrm{H} \cdots \mathrm{O}$ hydrogen bonds are shown as dashed lines. [Symmetry codes: (i) $-x,-y,-z$; (vi) $-x+3,-y,-z+1$.] 
Table 2

Hydrogen-bond geometry $\left(\AA,^{\circ}\right)$ for (II).

\begin{tabular}{lllll}
\hline$D-\mathrm{H} \cdots A$ & $D-\mathrm{H}$ & $\mathrm{H} \cdots A$ & $D \cdots A$ & $D-\mathrm{H} \cdots A$ \\
\hline $\mathrm{O} 3-\mathrm{H} 3 \cdots \mathrm{O} 2$ & $0.852(17)$ & $1.803(17)$ & $2.6277(12)$ & $162.5(16)$ \\
$\mathrm{O} 3-\mathrm{H} 3 \cdots \mathrm{O} 1^{\mathrm{i}}$ & $0.852(17)$ & $2.438(17)$ & $2.9738(12)$ & $121.6(14)$ \\
$\mathrm{N} 1-\mathrm{H} 1 \cdots \mathrm{O} 2^{\text {ii }}$ & $0.889(17)$ & $1.807(17)$ & $2.6684(12)$ & $162.6(16)$ \\
$\mathrm{C} 8-\mathrm{H} 8 \cdots \mathrm{O} 1^{\text {iii }}$ & 0.95 & 2.45 & $3.1481(13)$ & 130 \\
\hline
\end{tabular}

Symmetry codes: (i) $-x+2,-y+1,-z+1$; (ii) $x,-y+\frac{3}{2}, z-\frac{1}{2}$; (iii) $x-1, y, z-1$.

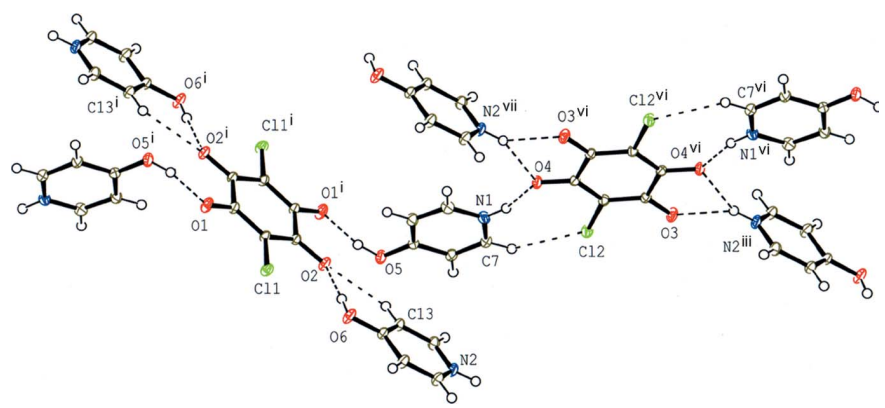

Figure 4

A partial packing diagram for compound (III) around two independent chloranilate anions. $\mathrm{O}-\mathrm{H} \cdots \mathrm{O}, \mathrm{N}-\mathrm{H} \cdots \mathrm{O}, \mathrm{C}-\mathrm{H} \cdots \mathrm{Cl}$ and $\mathrm{C}-\mathrm{H} \cdots \mathrm{O}$ hydrogen bonds are shown as dashed lines. [Symmetry codes: (i) $-x,-y$, $-z$; (iii) $-x+3,-y+1,-z+1$; (vi) $-x+3,-y,-z+1$; (vii) $x, y-1, z$.]

\section{Supramolecular features}

In the crystal of compound (I), two adjacent 2-pyridone molecules, which are related by a twofold rotation axis, form a head-to-head dimer via a pair of $\mathrm{N}-\mathrm{H} \cdots \mathrm{O}$ hydrogen bonds $\left(\mathrm{N} 1-\mathrm{H} 1 \cdots \mathrm{O} 3^{\mathrm{i}}\right.$; symmetry code as in Table 1$)$, as observed in various cocrystals of 2-pyridone (Odani \& Matsumoto, 2002).

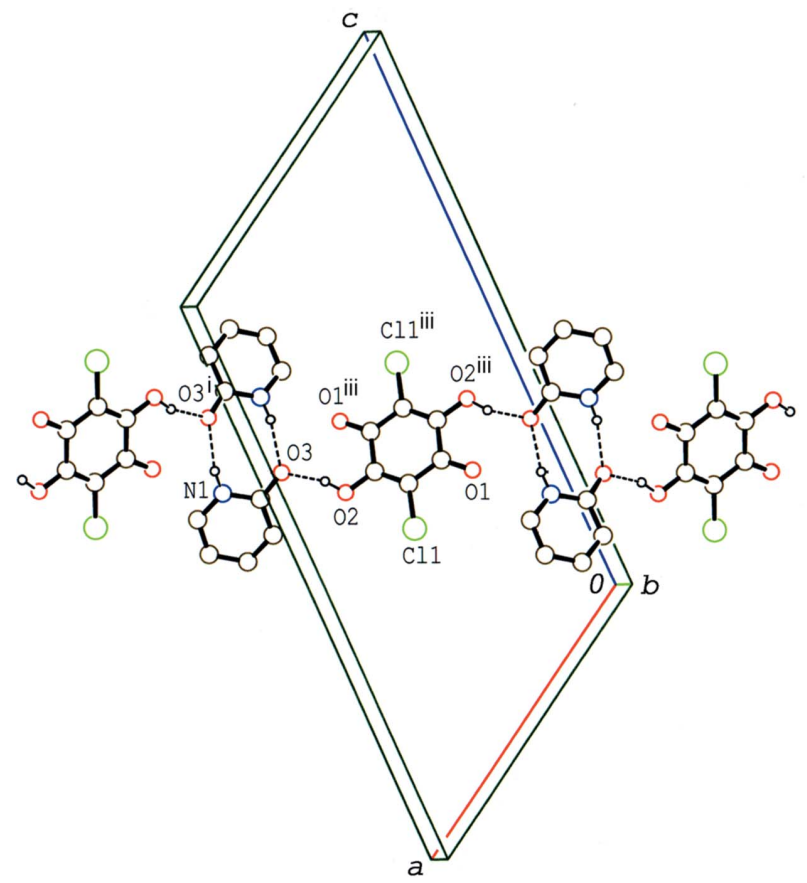

Figure 5

A packing diagram for compound (I), showing the tape structure formed by $\mathrm{O}-\mathrm{H} \cdots \mathrm{O}$ and $\mathrm{N}-\mathrm{H} \cdots \mathrm{O}$ hydrogen bonds (dashed lines). $\mathrm{H}$ atoms not involved in the interactions have been omitted. [Symmetry codes: (i) $-x+2, y,-z+\frac{3}{2}$; (iii) $-x+1,-y+1,-z+1$.]
Table 3

Hydrogen-bond geometry $\left(\AA{ }^{\circ}\right)$ for (III).

\begin{tabular}{lllll}
\hline$D-\mathrm{H} \cdots A$ & $D-\mathrm{H}$ & $\mathrm{H} \cdots A$ & $D \cdots A$ & $D-\mathrm{H} \cdots A$ \\
\hline $\mathrm{O} 5-\mathrm{H} 5 \cdots \mathrm{O} 1^{\mathrm{i}}$ & $0.905(17)$ & $1.640(17)$ & $2.5208(13)$ & $163.2(17)$ \\
$\mathrm{O} 6-\mathrm{H} 6 \cdots \mathrm{O} 2$ & $0.852(17)$ & $1.800(17)$ & $2.6510(13)$ & $177.3(18)$ \\
$\mathrm{N} 1-\mathrm{H} 1 \cdots \mathrm{O} 4$ & $0.919(17)$ & $1.810(17)$ & $2.7000(13)$ & $162.3(16)$ \\
$\mathrm{N} 2-\mathrm{H} 2 \cdots \mathrm{O} 4^{\mathrm{ii}}$ & $0.876(18)$ & $2.156(17)$ & $2.9603(14)$ & $152.3(15)$ \\
$\mathrm{N} 2-\mathrm{H} 2 \cdots 3^{\mathrm{iii}}$ & $0.876(18)$ & $2.176(17)$ & $2.8384(14)$ & $132.1(14)$ \\
$\mathrm{C} 7-\mathrm{H} 7 \cdots \mathrm{Cl} 2$ & 0.95 & 2.81 & $3.4540(12)$ & 126 \\
$\mathrm{C} 12-\mathrm{H} 12 \cdots \mathrm{O} 3^{\text {iv }}$ & 0.95 & 2.32 & $3.1541(14)$ & 146 \\
$\mathrm{C} 13-\mathrm{H} 13 \cdots \mathrm{O} 2$ & 0.95 & 2.49 & $3.1685(14)$ & 128 \\
$\mathrm{C}^{2} 6-\mathrm{H} 16 \cdots \mathrm{Cl}^{\mathrm{v}}$ & 0.95 & 2.77 & $3.4427(12)$ & 128 \\
\hline
\end{tabular}

Symmetry codes: (i) $-x,-y,-z$; (ii) $x, y+1, z$; (iii) $-x+3,-y+1,-z+1$; (iv) $-x+2,-y+1,-z+1 ;(\mathrm{v})-x+2,-y+1,-z$.

The acid and base molecules form an undulating tape structure running along [201] through the above-mentioned $\mathrm{O}-$ $\mathrm{H} \cdots \mathrm{O}$ and $\mathrm{N}-\mathrm{H} \cdots \mathrm{O}$ hydrogen bonds (Fig. 5). The tapes are stacked along the $b$ axis into a layer structure through a $\pi-\pi$ interaction between the pyridine rings [centroid-to-centroid distance $=3.7005(6) \AA$ and interplanar spacing $=$ $3.4239(4) \AA]$ and a short $\mathrm{C} \cdots \mathrm{C}$ contact $\left[\mathrm{C} 2 \cdots \mathrm{C} 3^{\text {iv }}=\right.$ 3.3056 (13) $\AA$; symmetry code: (iv) $x, y+1, z]$. A weak $\mathrm{C}-$ $\mathrm{H} \cdots \mathrm{Cl}$ interaction formed between the acid and base molecules $\left(\mathrm{C} 7-\mathrm{H} 7 \cdots \mathrm{Cl}^{\mathrm{ii}}\right.$; Table 1$)$ links the layers. The $\mathrm{O}-$ $\mathrm{H}$... O hydrogen bond between the acid and base molecules is short $[\mathrm{O} 2 \cdots \mathrm{O} 3=2.4989$ (11) $\AA$ ], suggesting possible disorder of the $\mathrm{H}$ atom in the hydrogen bond, but no distinct evidence of the disorder was observed in the difference Fourier map, nor from the molecular geometry.

In the crystal of (II), the cation-anion units are further connected by $\mathrm{N}-\mathrm{H} \cdots \mathrm{O}\left(\mathrm{N} 1-\mathrm{H} 1 \cdots \mathrm{O} 2^{\mathrm{ii}}\right.$; symmetry code as in Table 2 and Fig. 6), forming a layer expanding parallel to the $b c$ plane (Fig. 7). Adjacent layers are connected to each other with a $\mathrm{C}-\mathrm{H} \cdots \mathrm{O}$ hydrogen bond $\left(\mathrm{C} 8-\mathrm{H} 8 \cdots \mathrm{O} 1^{\mathrm{iii}}\right.$; Table 2$)$ and a short $\mathrm{O} \cdots \mathrm{N}$ contact $\left[\mathrm{O} 3 \cdots \mathrm{N} 1^{\mathrm{vi}}=3.0430(12) \AA\right.$ symmetry code: (vi) $\left.-x+1, y-\frac{1}{2},-z+\frac{1}{2}\right]$.

In the crystal of (III), the above-mentioned $\mathrm{O}-\mathrm{H} \cdots \mathrm{O}, \mathrm{N}-$ $\mathrm{H} \cdots \mathrm{O}, \mathrm{C}-\mathrm{H} \cdots \mathrm{O}$ and $\mathrm{C}-\mathrm{H} \cdots \mathrm{Cl}$ hydrogen bonds link the

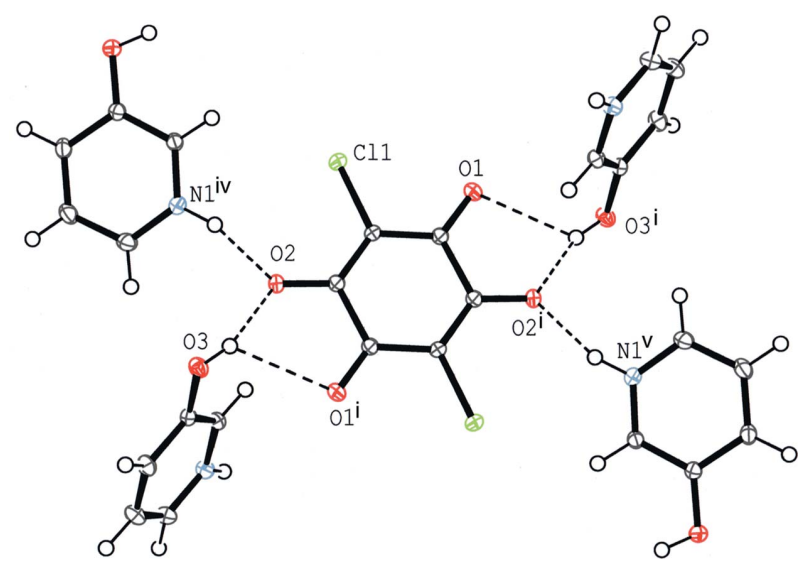

Figure 6

A partial packing diagram for compound (II) around the chloranilate anion. $\mathrm{O}-\mathrm{H} \cdots \mathrm{O}$ and $\mathrm{N}-\mathrm{H} \cdots \mathrm{O}$ hydrogen bonds are shown as dashed lines. [Symmetry codes: (i) $-x+2,-y+1,-z+1$; (iv) $x,-y+\frac{3}{2}, z+\frac{1}{2}$; (v) $-x+2, y-\frac{1}{2},-z+\frac{1}{2}$.] 
Table 4

Experimental details.

(I)

Crystal data

Chemical formula

$M_{\mathrm{r}}$

Crystal system, space group

Temperature (K)

$a, b, c(\AA)$

$\alpha, \beta, \gamma\left({ }^{\circ}\right)$

$V\left(\AA^{3}\right)$

$Z$

Radiation type

$\mu\left(\mathrm{mm}^{-1}\right)$

Crystal size (mm)

Data collection

Diffractometer

Absorption correction

$T_{\min }, T_{\max }$

No. of measured, independent and observed $[I>2 \sigma(I)]$ reflections

$R_{\text {int }}$

$(\sin \theta / \lambda)_{\max }\left(\AA^{-1}\right)$

0.013
0.703

Refinement

$R\left[F^{2}>2 \sigma\left(F^{2}\right)\right], w R\left(F^{2}\right), S$

No. of reflections

No. of parameters

$\mathrm{H}$-atom treatment

$\Delta \rho_{\max }, \Delta \rho_{\min }\left(\mathrm{e} \AA^{-3}\right)$
0.703

\author{
$2 \mathrm{C}_{5} \mathrm{H}_{5} \mathrm{NO} \cdot \mathrm{C}_{6} \mathrm{H}_{2} \mathrm{Cl}_{2} \mathrm{O}_{4}$ \\ 399.19 \\ Monoclinic, $P 2 / c$ \\ 120 \\ 11.9402 (7), 3.7005 (2), \\ 21.7919 (13) \\ 90, $121.278(2), 90$ \\ $822.92(9)$ \\ 2 \\ Mo $K \alpha$ \\ 0.43 \\ $0.39 \times 0.36 \times 0.21$
}

Rigaku R-AXIS RAPIDII

Multi-scan (ABSCOR; Higashi, 1995)

$0.804,0.913$

$22767,2401,2316$

$0.027,0.076,1.06$

2401

124

$\mathrm{H}$ atoms treated by a mixture of independent and constrained refinement

$0.49,-0.24$
(II)

$2 \mathrm{C}_{5} \mathrm{H}_{6} \mathrm{NO}^{+} \cdot \mathrm{C}_{6} \mathrm{Cl}_{2} \mathrm{O}_{4}{ }^{2-}$
399.19
Monoclinic, $P 2_{1} / c$
120
$8.3659(6), 8.5492(6), 11.7087(8)$
$90,106.968(3), 90$
$800.98(9)$
2
Mo $K \alpha$
0.45
$0.21 \times 0.20 \times 0.12$

Rigaku R-AXIS RAPIDII

Numerical (NUMABS; Higashi, 1999)

$0.903,0.948$

$15315,2329,2166$

0.017

0.703

$0.028,0.075,1.07$

2329

124

$\mathrm{H}$ atoms treated by a mixture of independent and constrained refinement

$0.52,-0.20$
(III)

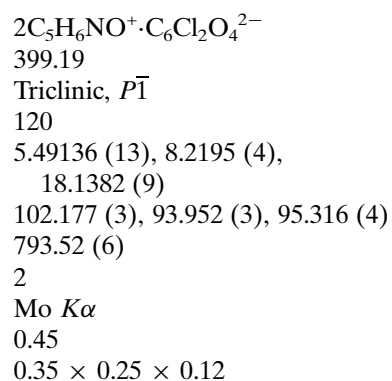

Rigaku R-AXIS RAPIDII

Numerical (NUMABS; Higashi, 1999)

$0.890,0.948$

$12373,4597,4124$

0.036

0.703

$0.030,0.080,1.07$

4597

247

$\mathrm{H}$ atoms treated by a mixture of independent and constrained refinement

$0.75,-0.34$

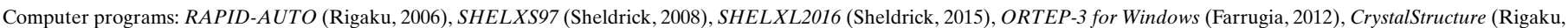
2010) and PLATON (Spek, 2009).

cations and anions into a layer parallel to (301) (Fig. 8). Adjacent layers are further linked via weak $\mathrm{C}-\mathrm{H} \cdots \mathrm{O}$ and $\mathrm{C}-\mathrm{H} \cdots \mathrm{Cl}$ interactions $\left(\mathrm{C} 12-\mathrm{H} 12 \cdots \mathrm{O}^{\mathrm{iv}}\right.$ and $\mathrm{C} 16-$ $\mathrm{H} 16 \cdot \mathrm{Cl1}^{\mathrm{v}}$; symmetry codes as given in Table 3 ).

\section{Database survey}

A search of the Cambridge Structural Database (Version 5.38, last update May 2017; Groom et al., 2016) for organic crystals of chloranilic acid with substituted pyridines (except for di-, tri- and tetrapyridine derivatives) gave 32 hits. Of these, crystal structures of 16 compounds of chloranilic acid with methyl-substituted pyridines (Adam et al., 2010; Łuczyńska et al., 2016; Molčanov \& Kojić-Prodić, 2010, and references therein), three compounds of carbamoyl-substituted pyridines (Gotoh et al., 2009a), three compounds of carboxy-substituted pyridines (Gotoh et al., 2009b, and references therein) and three compounds of cyano-substituted pyridines (Gotoh \& Ishida, 2012, and references therein) were reported.

\section{Synthesis and crystallization}

Single crystals of compound (I) were obtained by slow evaporation from an ethanol solution $(120 \mathrm{ml})$ of chloranilic acid (350 mg) with 2-hydroxypyridine $(340 \mathrm{mg}$ ) at room temperature. Crystals of compound (II) were obtained by slow evaporation from a methanol solution $(400 \mathrm{ml})$ of chloranilic acid $(170 \mathrm{mg})$ with 3-hydroxypyridine $(160 \mathrm{mg})$ at room temperature. Crystals of compound (III) were obtained by slow diffusion of a methanol solution $(20 \mathrm{ml})$ of 4-hydroxypyridine $(160 \mathrm{mg})$ into an acetonitrile solution $(200 \mathrm{ml})$ of chloranilic acid $(170 \mathrm{mg})$ at room temperature.

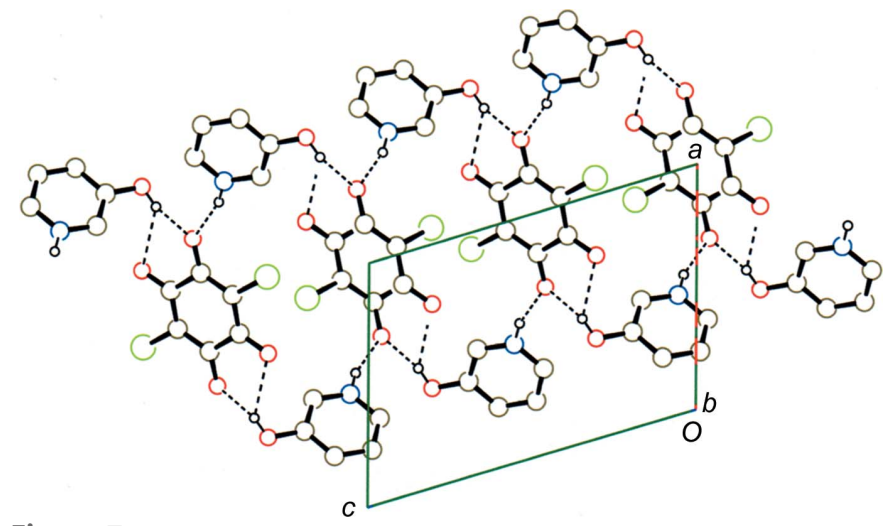

Figure 7

A packing diagram for compound (II), viewed along the $b$ axis, showing the layer structure formed by $\mathrm{O}-\mathrm{H} \cdots \mathrm{O}$ and $\mathrm{N}-\mathrm{H} \cdots \mathrm{O}$ hydrogen bonds (dashed lines). $\mathrm{H}$ atoms not involved in the interactions have been omitted. 


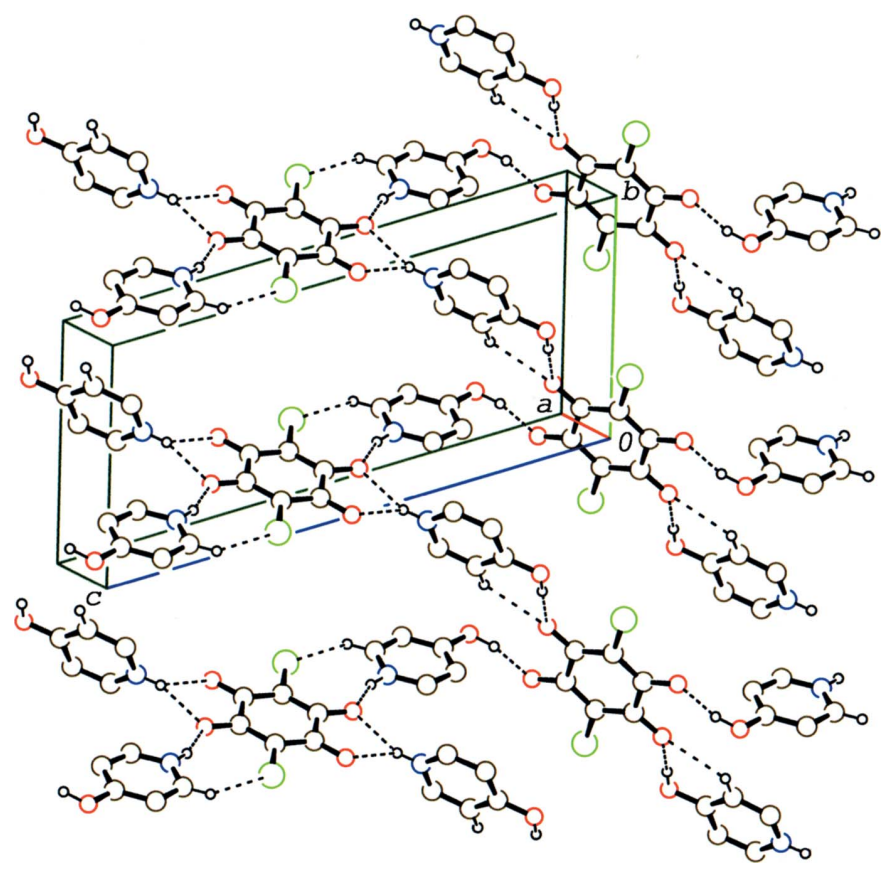

Figure 8

A packing diagram for compound (III), showing the hydrogen-bonded network in the layer. $\mathrm{O}-\mathrm{H} \cdots \mathrm{O}, \mathrm{N}-\mathrm{H} \cdots \mathrm{O}, \mathrm{C}-\mathrm{H} \cdots \mathrm{Cl}$ and $\mathrm{C}-\mathrm{H} \cdots \mathrm{O}$ hydrogen bonds are shown as dashed lines. $\mathrm{H}$ atoms not involved in the hydrogen bonds have been omitted.

\section{Refinement}

Crystal data, data collection and structure refinement details are summarized in Table 4. All $\mathrm{H}$ atoms in compounds (I)(III) were found in difference Fourier maps. The positions of
O- and $\mathrm{N}$-bound $\mathrm{H}$ atoms were refined freely, with $U_{\text {iso }}(\mathrm{H})=$ $1.5 U_{\text {eq }}(\mathrm{O}$ or $\mathrm{N})$. C-bound $\mathrm{H}$ atoms were positioned geometrically $(\mathrm{C}-\mathrm{H}=0.95 \AA)$ and were treated as riding with $U_{\text {iso }}(\mathrm{H})=1.2 U_{\text {eq }}(\mathrm{C})$.

\section{References}

Adam, M. S., Parkin, A., Thomas, L. H. \& Wilson, C. C. (2010). CrystEngComm, 12, 917-924.

Asaji, T., Seliger, J., Žagar, V. \& Ishida, H. (2010). Magn. Reson. Chem. 48, 531-536.

Farrugia, L. J. (2012). J. Appl. Cryst. 45, 849-854.

Gotoh, K., Asaji, T. \& Ishida, H. (2010). Acta Cryst. C66, o114-o118.

Gotoh, K. \& Ishida, H. (2009). Acta Cryst. E65, o3060.

Gotoh, K. \& Ishida, H. (2012). Acta Cryst. E68, o2830.

Gotoh, K., Nagoshi, H. \& Ishida, H. (2009a). Acta Cryst. C65, o273o277.

Gotoh, K., Nagoshi, H. \& Ishida, H. (2009b). Acta Cryst. E65, o614.

Groom, C. R., Bruno, I. J., Lightfoot, M. P. \& Ward, S. C. (2016). Acta Cryst. B72, 171-179.

Higashi, T. (1995). ABCSOR. Rigaku Corporation, Tokyo, Japan.

Higashi, T. (1999). NUMABS. Rigaku Corporation, Tokyo, Japan.

Łuczyńska, K., Drużbicki, K., Lyczko, K. \& Dobrowolski, J. Cz. (2016). Cryst. Growth Des. 16, 6069-6083.

Molčanov, K. \& Kojić-Prodić, B. (2010). CrystEngComm, 12, 925-939. Odani, T. \& Matsumoto, A. (2002). CrystEngComm, 4, 467-471.

Rigaku (2006). RAPID-AUTO. Rigaku Corporation, Tokyo, Japan. Rigaku (2010). CrystalStructure. Rigaku Corporation, Tokyo, Japan. Seliger, J., Žagar, V., Gotoh, K., Ishida, H., Konnai, A., Amino, D. \& Asaji, T. (2009). Phys. Chem. Chem. Phys. 11, 2281-2286.

Sheldrick, G. M. (2008). Acta Cryst. A64, 112-122.

Sheldrick, G. M. (2015). Acta Cryst. C71, 3-8.

Spek, A. L. (2009). Acta Cryst. D65, 148-155.

Zaman, Md. B., Udachin, K. A. \& Ripmeester, J. A. (2004). Cryst. Growth Des. 4, 585-589. 


\section{supporting information}

Acta Cryst. (2017). E73, 1546-1550 [https://doi.org/10.1107/S2056989017013536]

Crystal structures of three hydrogen-bonded 1:2 compounds of chloranilic acid with 2-pyridone, 3-hydroxypyridine and 4-hyroxypyridine

\section{Kazuma Gotoh and Hiroyuki Ishida}

\section{Computing details}

For all structures, data collection: RAPID-AUTO (Rigaku, 2006); cell refinement: RAPID-AUTO (Rigaku, 2006); data reduction: RAPID-AUTO (Rigaku, 2006); program(s) used to solve structure: SHELXS97 (Sheldrick, 2008); program(s) used to refine structure: SHELXL2016 (Sheldrick, 2015); molecular graphics: ORTEP-3 for Windows (Farrugia, 2012). Software used to prepare material for publication: CrystalStructure (Rigaku, 2010), PLATON (Spek, 2009) for (I); CrystalStructure (Rigaku, 2010) and PLATON (Spek, 2009) for (II), (III).

Bis[pyridin-2(1H)-one] 2,5-dichloro-3,6-dihydroxy-1,4-bebzoquinone (I)

Crystal data

$2 \mathrm{C}_{5} \mathrm{H}_{5} \mathrm{NO} \cdot \mathrm{C}_{6} \mathrm{H}_{2} \mathrm{Cl}_{2} \mathrm{O}_{4}$

$M_{r}=399.19$

Monoclinic, $P 2 / c$

$a=11.9402(7) \AA$

$b=3.7005(2) \AA$

$c=21.7919(13) \AA$

$\beta=121.278(2)^{\circ}$

$V=822.92(9) \AA^{3}$

$Z=2$

Data collection

Rigaku R-AXIS RAPIDII diffractometer

Detector resolution: 10.000 pixels $\mathrm{mm}^{-1}$

$\omega$ scans

Absorption correction: multi-scan

(ABSCOR; Higashi, 1995)

$T_{\text {min }}=0.804, T_{\max }=0.913$

22767 measured reflections

\section{Refinement}

Refinement on $F^{2}$

Least-squares matrix: full

$R\left[F^{2}>2 \sigma\left(F^{2}\right)\right]=0.027$

$w R\left(F^{2}\right)=0.076$

$S=1.06$

2401 reflections

124 parameters

0 restraints
$F(000)=408.00$

$D_{\mathrm{x}}=1.611 \mathrm{Mg} \mathrm{m}^{-3}$

Mo $K \alpha$ radiation, $\lambda=0.71075 \AA$

Cell parameters from 20111 reflections

$\theta=3.3-30.1^{\circ}$

$\mu=0.43 \mathrm{~mm}^{-1}$

$T=120 \mathrm{~K}$

Block, brown

$0.39 \times 0.36 \times 0.21 \mathrm{~mm}$

2401 independent reflections

2316 reflections with $I>2 \sigma(I)$

$R_{\text {int }}=0.013$

$\theta_{\text {max }}=30.0^{\circ}, \theta_{\min }=3.4^{\circ}$

$h=-16 \rightarrow 16$

$k=-4 \rightarrow 5$

$l=-30 \rightarrow 30$

Primary atom site location: structure-invariant direct methods

Secondary atom site location: difference Fourier map

Hydrogen site location: mixed

$\mathrm{H}$ atoms treated by a mixture of independent and constrained refinement 
$w=1 /\left[\sigma^{2}\left(F_{\mathrm{o}}^{2}\right)+(0.0443 P)^{2}+0.3557 P\right]$

where $P=\left(F_{\mathrm{o}}^{2}+2 F_{\mathrm{c}}{ }^{2}\right) / 3$

$(\Delta / \sigma)_{\max }=0.001$

$$
\Delta \rho_{\max }=0.49 \text { e } \AA^{-3}
$$

\section{Special details}

Geometry. All esds (except the esd in the dihedral angle between two 1.s. planes) are estimated using the full covariance matrix. The cell esds are taken into account individually in the estimation of esds in distances, angles and torsion angles; correlations between esds in cell parameters are only used when they are defined by crystal symmetry. An approximate (isotropic) treatment of cell esds is used for estimating esds involving 1.s. planes.

Fractional atomic coordinates and isotropic or equivalent isotropic displacement parameters $\left(\AA^{2}\right)$

\begin{tabular}{lllll}
\hline & $x$ & $y$ & $z$ & $U_{\text {iso }} * / U_{\mathrm{eq}}$ \\
\hline C11 & $0.60869(2)$ & $0.78653(6)$ & $0.40403(2)$ & $0.01704(8)$ \\
O1 & $0.33921(7)$ & $0.8229(2)$ & $0.37552(4)$ & $0.02149(16)$ \\
O2 & $0.75999(6)$ & $0.4050(2)$ & $0.54453(4)$ & $0.01794(15)$ \\
H2 & $0.8039(15)$ & $0.289(4)$ & $0.5870(8)$ & $0.027^{*}$ \\
O3 & $0.92219(7)$ & $0.1199(2)$ & $0.66164(4)$ & $0.02094(16)$ \\
N1 & $1.13199(8)$ & $-0.0736(2)$ & $0.72858(4)$ & $0.01604(16)$ \\
H1 & $1.1281(13)$ & $-0.016(4)$ & $0.7672(8)$ & $0.024 *$ \\
C1 & $0.41591(9)$ & $0.6765(2)$ & $0.43248(5)$ & $0.01361(17)$ \\
C2 & $0.55361(8)$ & $0.6274(3)$ & $0.45806(5)$ & $0.01311(16)$ \\
C3 & $0.63639(8)$ & $0.4587(2)$ & $0.52136(5)$ & $0.01342(17)$ \\
C4 & $1.02174(9)$ & $-0.0153(3)$ & $0.66317(5)$ & $0.01563(17)$ \\
C5 & $1.02800(9)$ & $-0.1116(3)$ & $0.60168(5)$ & $0.01779(18)$ \\
H5 & 0.953363 & -0.080221 & 0.554948 & $0.021 *$ \\
C6 & $1.14171(10)$ & $-0.2497(3)$ & $0.60990(5)$ & $0.01837(19)$ \\
H6 & 1.145376 & -0.312374 & 0.568705 & $0.022 *$ \\
C7 & $1.25330(10)$ & $-0.2995(3)$ & $0.67902(5)$ & $0.01870(19)$ \\
H7 & 1.332337 & -0.392760 & 0.684778 & $0.022^{*}$ \\
C8 & $1.24515(9)$ & $-0.2109(3)$ & $0.73732(5)$ & $0.01775(18)$ \\
H8 & 1.318846 & -0.245048 & 0.784277 & $0.021 *$
\end{tabular}

Atomic displacement parameters $\left(\AA^{2}\right)$

\begin{tabular}{lllllll}
\hline & $U^{11}$ & $U^{22}$ & $U^{33}$ & $U^{12}$ & $U^{13}$ & $U^{23}$ \\
\hline C11 & $0.02123(12)$ & $0.01778(12)$ & $0.01883(12)$ & $0.00121(8)$ & $0.01512(10)$ & $0.00259(7)$ \\
O1 & $0.0172(3)$ & $0.0304(4)$ & $0.0166(3)$ & $0.0046(3)$ & $0.0086(3)$ & $0.0080(3)$ \\
O2 & $0.0124(3)$ & $0.0244(4)$ & $0.0172(3)$ & $0.0022(3)$ & $0.0079(2)$ & $0.0040(3)$ \\
O3 & $0.0149(3)$ & $0.0307(4)$ & $0.0184(3)$ & $0.0048(3)$ & $0.0095(3)$ & $0.0034(3)$ \\
N1 & $0.0158(3)$ & $0.0189(4)$ & $0.0145(3)$ & $0.0021(3)$ & $0.0086(3)$ & $0.0000(3)$ \\
C1 & $0.0150(4)$ & $0.0138(4)$ & $0.0134(4)$ & $0.0000(3)$ & $0.0083(3)$ & $-0.0005(3)$ \\
C2 & $0.0150(4)$ & $0.0139(4)$ & $0.0139(4)$ & $0.0000(3)$ & $0.0098(3)$ & $0.0002(3)$ \\
C3 & $0.0139(4)$ & $0.0137(4)$ & $0.0143(4)$ & $-0.0005(3)$ & $0.0084(3)$ & $-0.0010(3)$ \\
C4 & $0.0142(4)$ & $0.0170(4)$ & $0.0162(4)$ & $-0.0005(3)$ & $0.0083(3)$ & $0.0012(3)$ \\
C5 & $0.0178(4)$ & $0.0207(4)$ & $0.0146(4)$ & $-0.0011(3)$ & $0.0082(3)$ & $0.0004(3)$ \\
C6 & $0.0221(4)$ & $0.0189(4)$ & $0.0179(4)$ & $-0.0015(3)$ & $0.0131(4)$ & $-0.0018(3)$ \\
C7 & $0.0184(4)$ & $0.0190(4)$ & $0.0211(4)$ & $0.0023(3)$ & $0.0119(4)$ & $-0.0009(3)$ \\
C8 & $0.0157(4)$ & $0.0191(4)$ & $0.0174(4)$ & $0.0033(3)$ & $0.0078(3)$ & $0.0002(3)$ \\
\hline
\end{tabular}


Geometric parameters $\left(\AA,{ }^{\circ}\right)$

\begin{tabular}{|c|c|c|c|}
\hline $\mathrm{C} 11-\mathrm{C} 2$ & $1.7233(9)$ & $\mathrm{C} 2-\mathrm{C} 3$ & $1.3614(12)$ \\
\hline $\mathrm{O} 1-\mathrm{C} 1$ & $1.2217(11)$ & $\mathrm{C} 4-\mathrm{C} 5$ & $1.4256(13)$ \\
\hline $\mathrm{O} 2-\mathrm{C} 3$ & $1.3033(10)$ & $\mathrm{C} 5-\mathrm{C} 6$ & $1.3726(14)$ \\
\hline $\mathrm{O} 2-\mathrm{H} 2$ & $0.901(16)$ & $\mathrm{C} 5-\mathrm{H} 5$ & 0.9500 \\
\hline $\mathrm{O} 3-\mathrm{C} 4$ & $1.2739(11)$ & $\mathrm{C} 6-\mathrm{C} 7$ & $1.4120(14)$ \\
\hline $\mathrm{N} 1-\mathrm{C} 8$ & $1.3611(12)$ & $\mathrm{C} 6-\mathrm{H} 6$ & 0.9500 \\
\hline $\mathrm{N} 1-\mathrm{C} 4$ & $1.3648(11)$ & $\mathrm{C} 7-\mathrm{C} 8$ & $1.3636(14)$ \\
\hline $\mathrm{N} 1-\mathrm{H} 1$ & $0.892(15)$ & $\mathrm{C} 7-\mathrm{H} 7$ & 0.9500 \\
\hline $\mathrm{C} 1-\mathrm{C} 2$ & $1.4475(12)$ & $\mathrm{C} 8-\mathrm{H} 8$ & 0.9500 \\
\hline $\mathrm{C} 1-\mathrm{C} 3^{\mathrm{i}}$ & $1.5182(12)$ & & \\
\hline $\mathrm{C} 3-\mathrm{O} 2-\mathrm{H} 2$ & $114.1(10)$ & $\mathrm{O} 3-\mathrm{C} 4-\mathrm{C} 5$ & $125.24(8)$ \\
\hline $\mathrm{C} 8-\mathrm{N} 1-\mathrm{C} 4$ & $123.66(8)$ & $\mathrm{N} 1-\mathrm{C} 4-\mathrm{C} 5$ & $116.69(8)$ \\
\hline $\mathrm{C} 8-\mathrm{N} 1-\mathrm{H} 1$ & $119.5(9)$ & $\mathrm{C} 6-\mathrm{C} 5-\mathrm{C} 4$ & $120.10(9)$ \\
\hline $\mathrm{C} 4-\mathrm{N} 1-\mathrm{H} 1$ & $116.9(9)$ & $\mathrm{C} 6-\mathrm{C} 5-\mathrm{H} 5$ & 120.0 \\
\hline $\mathrm{O} 1-\mathrm{C} 1-\mathrm{C} 2$ & $123.32(8)$ & $\mathrm{C} 4-\mathrm{C} 5-\mathrm{H} 5$ & 120.0 \\
\hline $\mathrm{O} 1-\mathrm{C} 1-\mathrm{C} 3^{\mathrm{i}}$ & $118.05(8)$ & $\mathrm{C} 5-\mathrm{C} 6-\mathrm{C} 7$ & $120.62(9)$ \\
\hline $\mathrm{C} 2-\mathrm{C} 1-\mathrm{C} 3^{\mathrm{i}}$ & $118.63(7)$ & $\mathrm{C} 5-\mathrm{C} 6-\mathrm{H} 6$ & 119.7 \\
\hline $\mathrm{C} 3-\mathrm{C} 2-\mathrm{C} 1$ & $121.99(8)$ & $\mathrm{C} 7-\mathrm{C} 6-\mathrm{H} 6$ & 119.7 \\
\hline $\mathrm{C} 3-\mathrm{C} 2-\mathrm{C} 11$ & $121.00(7)$ & $\mathrm{C} 8-\mathrm{C} 7-\mathrm{C} 6$ & $118.57(9)$ \\
\hline $\mathrm{C} 1-\mathrm{C} 2-\mathrm{C} 11$ & $117.01(7)$ & $\mathrm{C} 8-\mathrm{C} 7-\mathrm{H} 7$ & 120.7 \\
\hline $\mathrm{O} 2-\mathrm{C} 3-\mathrm{C} 2$ & $122.88(8)$ & $\mathrm{C} 6-\mathrm{C} 7-\mathrm{H} 7$ & 120.7 \\
\hline $\mathrm{O} 2-\mathrm{C} 3-\mathrm{C}^{\mathrm{i}}$ & $117.75(8)$ & $\mathrm{N} 1-\mathrm{C} 8-\mathrm{C} 7$ & $120.35(9)$ \\
\hline $\mathrm{C} 2-\mathrm{C} 3-\mathrm{C}^{\mathrm{i}}$ & $119.37(8)$ & $\mathrm{N} 1-\mathrm{C} 8-\mathrm{H} 8$ & 119.8 \\
\hline $\mathrm{O} 3-\mathrm{C} 4-\mathrm{N} 1$ & $118.06(8)$ & $\mathrm{C} 7-\mathrm{C} 8-\mathrm{H} 8$ & 119.8 \\
\hline $\mathrm{O} 1-\mathrm{C} 1-\mathrm{C} 2-\mathrm{C} 3$ & $-179.08(9)$ & $\mathrm{C} 8-\mathrm{N} 1-\mathrm{C} 4-\mathrm{O} 3$ & $-178.90(9)$ \\
\hline $\mathrm{C} 3-\mathrm{C} 1-\mathrm{C} 2-\mathrm{C} 3$ & $1.18(15)$ & $\mathrm{C} 8-\mathrm{N} 1-\mathrm{C} 4-\mathrm{C} 5$ & $1.04(15)$ \\
\hline $\mathrm{O} 1-\mathrm{C} 1-\mathrm{C} 2-\mathrm{C} 11$ & $-0.05(13)$ & $\mathrm{O} 3-\mathrm{C} 4-\mathrm{C} 5-\mathrm{C} 6$ & $178.84(10)$ \\
\hline $\mathrm{C} 3-\mathrm{C} 1-\mathrm{C} 2-\mathrm{C} 11$ & $-179.79(6)$ & $\mathrm{N} 1-\mathrm{C} 4-\mathrm{C} 5-\mathrm{C} 6$ & $-1.09(15)$ \\
\hline $\mathrm{C} 1-\mathrm{C} 2-\mathrm{C} 3-\mathrm{O} 2$ & $178.46(9)$ & $\mathrm{C} 4-\mathrm{C} 5-\mathrm{C} 6-\mathrm{C} 7$ & $0.28(16)$ \\
\hline $\mathrm{C} 11-\mathrm{C} 2-\mathrm{C} 3-\mathrm{O} 2$ & $-0.53(14)$ & $\mathrm{C} 5-\mathrm{C} 6-\mathrm{C} 7-\mathrm{C} 8$ & $0.64(16)$ \\
\hline $\mathrm{C} 1-\mathrm{C} 2-\mathrm{C} 3-\mathrm{C}^{\mathrm{i}}$ & $-1.19(15)$ & $\mathrm{C} 4-\mathrm{N} 1-\mathrm{C} 8-\mathrm{C} 7$ & $-0.13(16)$ \\
\hline $\mathrm{C} 11-\mathrm{C} 2-\mathrm{C} 3-\mathrm{C} 1^{\mathrm{i}}$ & $179.82(7)$ & $\mathrm{C} 6-\mathrm{C} 7-\mathrm{C} 8-\mathrm{N} 1$ & $-0.73(15)$ \\
\hline
\end{tabular}

Symmetry code: (i) $-x+1,-y+1,-z+1$.

Hydrogen-bond geometry $\left(A,{ }^{\circ}\right)$

\begin{tabular}{lllll}
\hline$D-\mathrm{H} \cdots A$ & $D-\mathrm{H}$ & $\mathrm{H} \cdots A$ & $D \cdots A$ & $D-\mathrm{H} \cdots A$ \\
\hline $\mathrm{O} 2-\mathrm{H} 2 \cdots \mathrm{O} 3$ & $0.901(15)$ & $1.627(16)$ & $2.4989(11)$ & $161.9(19)$ \\
$\mathrm{N} 1-\mathrm{H} 1 \cdots \mathrm{O} 3^{\mathrm{ii}}$ & $0.893(16)$ & $1.996(17)$ & $2.8743(12)$ & $167.6(16)$ \\
$\mathrm{C} 7-\mathrm{H} 7 \cdots \mathrm{Cl1}^{\mathrm{iii}}$ & 0.95 & 2.79 & $3.5122(13)$ & 134
\end{tabular}

Symmetry codes: (ii) $-x+2, y,-z+3 / 2$; (iii) $-x+2,-y,-z+1$. 
Bis(3-hyroxypyridinium) 2,5-dichloro-3,6-dioxocyclohexa-1,4-diene-1,4-diolate (II)

Crystal data

$2 \mathrm{C}_{5} \mathrm{H}_{6} \mathrm{NO}^{+} \cdot \mathrm{C}_{6} \mathrm{Cl}_{2} \mathrm{O}_{4}{ }^{2-}$

$M_{r}=399.19$

Monoclinic, $P 2_{1} / c$

$a=8.3659(6) \AA$

$b=8.5492(6) \AA$

$c=11.7087(8) \AA$

$\beta=106.968(3)^{\circ}$

$V=800.98(9) \AA^{3}$

$Z=2$

$F(000)=408.00$

$D_{\mathrm{x}}=1.655 \mathrm{Mg} \mathrm{m}^{-3}$

Mo $K \alpha$ radiation, $\lambda=0.71075 \AA$

Cell parameters from 13386 reflections

$\theta=3.0-30.0^{\circ}$

$\mu=0.45 \mathrm{~mm}^{-1}$

$T=120 \mathrm{~K}$

Block, brown

$0.21 \times 0.20 \times 0.12 \mathrm{~mm}$

\section{Data collection}

Rigaku R-AXIS RAPIDII diffractometer

Detector resolution: 10.000 pixels $\mathrm{mm}^{-1}$

$\omega$ scans

Absorption correction: numerical

(NUMABS; Higashi, 1999)

$T_{\min }=0.903, T_{\max }=0.948$

15315 measured reflections

\section{Refinement}

Refinement on $F^{2}$

Least-squares matrix: full

$R\left[F^{2}>2 \sigma\left(F^{2}\right)\right]=0.028$

$w R\left(F^{2}\right)=0.075$

$S=1.07$

2329 reflections

124 parameters

0 restraints

Primary atom site location: structure-invariant direct methods
2329 independent reflections

2166 reflections with $I>2 \sigma(I)$

$R_{\text {int }}=0.017$

$\theta_{\text {max }}=30.0^{\circ}, \theta_{\min }=3.0^{\circ}$

$h=-11 \rightarrow 11$

$k=-12 \rightarrow 12$

$l=-15 \rightarrow 16$
Secondary atom site location: difference Fourier map

Hydrogen site location: mixed

$\mathrm{H}$ atoms treated by a mixture of independent and constrained refinement

$w=1 /\left[\sigma^{2}\left(F_{\mathrm{o}}^{2}\right)+(0.0411 P)^{2}+0.357 P\right]$ where $P=\left(F_{\mathrm{o}}{ }^{2}+2 F_{\mathrm{c}}{ }^{2}\right) / 3$

$(\Delta / \sigma)_{\max }=0.001$

$\Delta \rho_{\max }=0.52 \mathrm{e} \AA^{-3}$

$\Delta \rho_{\min }=-0.20$ e $\AA^{-3}$

Special details

Geometry. All esds (except the esd in the dihedral angle between two 1.s. planes) are estimated using the full covariance matrix. The cell esds are taken into account individually in the estimation of esds in distances, angles and torsion angles; correlations between esds in cell parameters are only used when they are defined by crystal symmetry. An approximate (isotropic) treatment of cell esds is used for estimating esds involving l.s. planes.

Fractional atomic coordinates and isotropic or equivalent isotropic displacement parameters $\left(\AA^{2}\right)$

\begin{tabular}{lllll}
\hline & $x$ & $y$ & $z$ & $U_{\text {iso }} * / U_{\text {eq }}$ \\
\hline C11 & $0.93157(3)$ & $0.73912(3)$ & $0.68834(2)$ & $0.01680(8)$ \\
O1 & $1.24731(9)$ & $0.58136(9)$ & $0.69329(7)$ & $0.01740(16)$ \\
O2 & $0.68396(9)$ & $0.61601(9)$ & $0.46095(7)$ & $0.01689(16)$ \\
O3 & $0.41012(10)$ & $0.51422(9)$ & $0.30475(7)$ & $0.01640(16)$ \\
H3 & $0.509(2)$ & $0.5340(19)$ & $0.3482(15)$ & $0.025^{*}$ \\
N1 & $0.48820(11)$ & $0.71217(11)$ & $0.05541(8)$ & $0.01529(17)$ \\
H1 & $0.566(2)$ & $0.7718(19)$ & $0.0393(15)$ & $0.023^{*}$ \\
C1 & $1.13017(12)$ & $0.54789(11)$ & $0.60534(9)$ & $0.01283(18)$ \\
C2 & $0.96428(12)$ & $0.60823(12)$ & $0.58348(9)$ & $0.01358(18)$
\end{tabular}




\begin{tabular}{lllll} 
C3 & $0.83396(12)$ & $0.56708(12)$ & $0.48551(9)$ & $0.01327(18)$ \\
C4 & $0.51167(12)$ & $0.66180(12)$ & $0.16734(9)$ & $0.01383(18)$ \\
H4 & 0.609229 & 0.691448 & 0.228545 & $0.017^{*}$ \\
C5 & $0.39288(12)$ & $0.56593(11)$ & $0.19388(9)$ & $0.01309(18)$ \\
C6 & $0.25163(13)$ & $0.52430(13)$ & $0.10152(10)$ & $0.0171(2)$ \\
H6 & 0.169400 & 0.457667 & 0.116909 & $0.021^{*}$ \\
C7 & $0.23181(14)$ & $0.58069(13)$ & $-0.01295(10)$ & $0.0192(2)$ \\
H7 & 0.135178 & 0.553857 & -0.075971 & $0.023^{*}$ \\
C8 & $0.35290(14)$ & $0.67593(13)$ & $-0.03506(9)$ & $0.0180(2)$ \\
H8 & 0.340534 & 0.715121 & -0.113094 & $0.022^{*}$ \\
\hline
\end{tabular}

Atomic displacement parameters $\left(\AA^{2}\right)$

\begin{tabular}{lllllll}
\hline & $U^{11}$ & $U^{22}$ & $U^{33}$ & $U^{12}$ & $U^{13}$ & $U^{23}$ \\
\hline C11 & $0.01596(13)$ & $0.01889(13)$ & $0.01530(13)$ & $0.00248(8)$ & $0.00420(9)$ & $-0.00478(8)$ \\
O1 & $0.0143(3)$ & $0.0210(4)$ & $0.0144(3)$ & $0.0017(3)$ & $0.0004(3)$ & $-0.0030(3)$ \\
O2 & $0.0118(3)$ & $0.0224(4)$ & $0.0151(3)$ & $0.0047(3)$ & $0.0018(3)$ & $-0.0023(3)$ \\
O3 & $0.0141(3)$ & $0.0203(4)$ & $0.0142(3)$ & $-0.0010(3)$ & $0.0032(3)$ & $0.0034(3)$ \\
N1 & $0.0148(4)$ & $0.0160(4)$ & $0.0155(4)$ & $-0.0020(3)$ & $0.0050(3)$ & $0.0005(3)$ \\
C1 & $0.0129(4)$ & $0.0133(4)$ & $0.0121(4)$ & $0.0010(3)$ & $0.0034(3)$ & $0.0006(3)$ \\
C2 & $0.0134(4)$ & $0.0151(4)$ & $0.0122(4)$ & $0.0021(3)$ & $0.0036(3)$ & $-0.0021(3)$ \\
C3 & $0.0132(4)$ & $0.0144(4)$ & $0.0120(4)$ & $0.0016(3)$ & $0.0034(3)$ & $0.0006(3)$ \\
C4 & $0.0122(4)$ & $0.0142(4)$ & $0.0142(4)$ & $-0.0007(3)$ & $0.0025(3)$ & $0.0001(3)$ \\
C5 & $0.0121(4)$ & $0.0127(4)$ & $0.0144(4)$ & $0.0010(3)$ & $0.0037(3)$ & $0.0001(3)$ \\
C6 & $0.0129(4)$ & $0.0180(5)$ & $0.0194(5)$ & $-0.0033(3)$ & $0.0031(4)$ & $0.0000(4)$ \\
C7 & $0.0167(5)$ & $0.0209(5)$ & $0.0169(5)$ & $-0.0031(4)$ & $-0.0001(4)$ & $-0.0019(4)$ \\
C8 & $0.0198(5)$ & $0.0199(5)$ & $0.0132(4)$ & $-0.0014(4)$ & $0.0031(4)$ & $-0.0005(4)$ \\
& & & & & &
\end{tabular}

Geometric parameters $\left(\AA,^{\circ}\right)$

\begin{tabular}{llll}
\hline $\mathrm{C} 11-\mathrm{C} 2$ & $1.7409(10)$ & $\mathrm{C} 2-\mathrm{C} 3$ & $1.3778(13)$ \\
$\mathrm{O} 1-\mathrm{C} 1$ & $1.2302(12)$ & $\mathrm{C} 4-\mathrm{C} 5$ & $1.3915(13)$ \\
$\mathrm{O} 2-\mathrm{C} 3$ & $1.2735(12)$ & $\mathrm{C} 4-\mathrm{H} 4$ & 0.9500 \\
$\mathrm{O} 3-\mathrm{C} 5$ & $1.3387(12)$ & $\mathrm{C} 5-\mathrm{C} 6$ & $1.3952(14)$ \\
$\mathrm{O} 3-\mathrm{H} 3$ & $0.850(18)$ & $\mathrm{C} 6-\mathrm{C} 7$ & $1.3880(15)$ \\
$\mathrm{N} 1-\mathrm{C} 4$ & $1.3384(13)$ & $\mathrm{C} 6-\mathrm{H} 6$ & 0.9500 \\
$\mathrm{~N} 1-\mathrm{C} 8$ & $1.3414(14)$ & $\mathrm{C} 7-\mathrm{C} 8$ & $1.3818(15)$ \\
$\mathrm{N} 1-\mathrm{H} 1$ & $0.891(17)$ & $\mathrm{C} 7-\mathrm{H} 7$ & 0.9500 \\
$\mathrm{C} 1-\mathrm{C} 2$ & $1.4319(13)$ & $\mathrm{C} 8-\mathrm{H} 8$ & 0.9500 \\
$\mathrm{C} 1-\mathrm{C} 3$ & & \\
& $1.5409(14)$ & & 120.1 \\
$\mathrm{C} 5-\mathrm{O} 3-\mathrm{H} 3$ & & & 120.1 \\
$\mathrm{C} 4-\mathrm{N} 1-\mathrm{C} 8$ & $109.0(11)$ & $\mathrm{N} 1-\mathrm{C} 4-\mathrm{H} 4$ & $121.95(9)$ \\
$\mathrm{C} 4-\mathrm{N} 1-\mathrm{H} 1$ & $123.22(9)$ & $\mathrm{C} 5-\mathrm{C} 4-\mathrm{H} 4$ & $119.64(9)$ \\
$\mathrm{C} 8-\mathrm{N} 1-\mathrm{H} 1$ & $119.0(11)$ & $\mathrm{O} 3-\mathrm{C} 5-\mathrm{C} 4$ & $119.41(9)$ \\
$\mathrm{O} 1-\mathrm{C} 1-\mathrm{C} 2$ & $117.8(11)$ & $\mathrm{O} 3-\mathrm{C} 5-\mathrm{C} 6$ & 120.2 \\
$\mathrm{O} 1-\mathrm{C} 1-\mathrm{C} 3^{\mathrm{i}}$ & $124.03(9)$ & $\mathrm{C} 4-\mathrm{C} 5-\mathrm{C} 6$ & \\
$\mathrm{C} 2-\mathrm{C} 1-\mathrm{C} 3^{\mathrm{i}}$ & $117.27(8)$ & $\mathrm{C} 7-\mathrm{C} 6-\mathrm{C} 5$ & $\mathrm{C} 7-\mathrm{C} 6-\mathrm{H} 6$ \\
& $118.70(8)$ & &
\end{tabular}




$\begin{array}{llll}\mathrm{C} 3-\mathrm{C} 2-\mathrm{C} 1 & 123.17(9) & \mathrm{C} 5-\mathrm{C} 6-\mathrm{H} 6 & 120.2 \\ \mathrm{C} 3-\mathrm{C} 2-\mathrm{C} 11 & 120.15(7) & \mathrm{C} 8-\mathrm{C} 7-\mathrm{C} 6 & 119.88(9) \\ \mathrm{C} 1-\mathrm{C} 2-\mathrm{C} 11 & 116.69(7) & \mathrm{C} 8-\mathrm{C} 7-\mathrm{H} 7 & 120.1 \\ \mathrm{O} 2-\mathrm{C} 3-\mathrm{C} 2 & 126.33(9) & \mathrm{C} 6-\mathrm{C} 7-\mathrm{H} 7 & 120.1 \\ \mathrm{O} 2-\mathrm{C} 3-\mathrm{C} 1^{\mathrm{i}} & 115.54(8) & \mathrm{N} 1-\mathrm{C} 8-\mathrm{C} 7 & 118.94(9) \\ \mathrm{C} 2-\mathrm{C} 3-\mathrm{C} 1^{\mathrm{i}} & 118.13(8) & \mathrm{N} 1-\mathrm{C} 8-\mathrm{H} 8 & 120.5 \\ \mathrm{~N} 1-\mathrm{C} 4-\mathrm{C} 5 & 119.86(9) & \mathrm{C} 7-\mathrm{C} 8-\mathrm{H} 8 & \\ & & & 0.55(16) \\ \mathrm{O} 1-\mathrm{C} 1-\mathrm{C} 2-\mathrm{C} 3 & -178.76(10) & \mathrm{C} 8-\mathrm{N} 1-\mathrm{C} 4-\mathrm{C} 5 & -179.23(9) \\ \mathrm{C} 3-\mathrm{C} 1-\mathrm{C} 2-\mathrm{C} 3 & 0.78(16) & \mathrm{N} 1-\mathrm{C} 4-\mathrm{C} 5-\mathrm{O} 3 & 0.30(15) \\ \mathrm{O} 1-\mathrm{C} 1-\mathrm{C} 2-\mathrm{C} 1 & 1.08(14) & \mathrm{N} 1-\mathrm{C} 4-\mathrm{C} 5-\mathrm{C} 6 & 178.57(10) \\ \mathrm{C} 3-\mathrm{C} 1-\mathrm{C} 2-\mathrm{C} 11 & -179.38(7) & \mathrm{O} 3-\mathrm{C} 5-\mathrm{C} 6-\mathrm{C} 7 & -0.97(15) \\ \mathrm{C} 1-\mathrm{C} 2-\mathrm{C} 3-\mathrm{O} 2 & 179.87(10) & \mathrm{C} 4-\mathrm{C} 5-\mathrm{C} 6-\mathrm{C} 7 & 0.83(17) \\ \mathrm{C} 11-\mathrm{C} 2-\mathrm{C} 3-\mathrm{O} 2 & 0.03(15) & \mathrm{C} 5-\mathrm{C} 6-\mathrm{C} 7-\mathrm{C} 8 & -0.70(16) \\ \mathrm{C} 1-\mathrm{C} 2-\mathrm{C} 3-\mathrm{C} 1^{\mathrm{i}} & -0.78(16) & \mathrm{C} 4-\mathrm{N} 1-\mathrm{C} 8-\mathrm{C} 7 & -0.01(17) \\ \mathrm{C} 11-\mathrm{C} 2-\mathrm{C} 3-\mathrm{C} 1^{\mathrm{i}} & 179.39(7) & \mathrm{C} 6-\mathrm{C} 7-\mathrm{C} 8-\mathrm{N} 1 & \end{array}$

Symmetry code: (i) $-x+2,-y+1,-z+1$.

Hydrogen-bond geometry $\left(\AA,{ }^{\circ}\right)$

\begin{tabular}{lllll}
\hline$D-\mathrm{H} \cdots A$ & $D-\mathrm{H}$ & $\mathrm{H} \cdots A$ & $D \cdots A$ & $D-\mathrm{H} \cdots A$ \\
\hline $\mathrm{O} 3-\mathrm{H} 3 \cdots \mathrm{O} 2$ & $0.852(17)$ & $1.803(17)$ & $2.6277(12)$ & $162.5(16)$ \\
$\mathrm{O} 3-\mathrm{H} 3 \cdots \mathrm{O} 1^{\mathrm{i}}$ & $0.852(17)$ & $2.438(17)$ & $2.9738(12)$ & $121.6(14)$ \\
$\mathrm{N} 1-\mathrm{H} 1 \cdots \mathrm{O} 2^{\mathrm{ii}}$ & $0.889(17)$ & $1.807(17)$ & $2.6684(12)$ & $162.6(16)$ \\
$\mathrm{C} 8-\mathrm{H} 8 \cdots{ }^{\mathrm{iii}}$ & 0.95 & 2.45 & $3.1481(13)$ & 130 \\
\hline
\end{tabular}

Symmetry codes: (i) $-x+2,-y+1,-z+1$; (ii) $x,-y+3 / 2, z-1 / 2$; (iii) $x-1, y, z-1$.

Bis(4-hyroxypyridinium) 2,5-dichloro-3,6-dioxocyclohexa-1,4-diene-1,4-diolate (III)

Crystal data

$2 \mathrm{C}_{5} \mathrm{H}_{6} \mathrm{NO}^{+} \cdot \mathrm{C}_{6} \mathrm{Cl}_{2} \mathrm{O}_{4}{ }^{2-}$

$M_{r}=399.19$

Triclinic, $P \overline{1}$

$a=5.49136(13) \AA$

$b=8.2195(4) \AA$

$c=18.1382(9) \AA$

$\alpha=102.177(3)^{\circ}$

$\beta=93.952(3)^{\circ}$

$\gamma=95.316(4)^{\circ}$

$V=793.52(6) \AA^{3}$

\section{Data collection}

Rigaku R-AXIS RAPIDII diffractometer

Detector resolution: 10.000 pixels $\mathrm{mm}^{-1}$ $\omega$ scans

Absorption correction: numerical (NUMABS; Higashi, 1999)

$T_{\min }=0.890, T_{\max }=0.948$

12373 measured reflections
$Z=2$

$F(000)=408.00$

$D_{\mathrm{x}}=1.671 \mathrm{Mg} \mathrm{m}^{-3}$

Mo $K \alpha$ radiation, $\lambda=0.71075 \AA$

Cell parameters from 11077 reflections

$\theta=3.0-30.1^{\circ}$

$\mu=0.45 \mathrm{~mm}^{-1}$

$T=120 \mathrm{~K}$

Platelet, brown

$0.35 \times 0.25 \times 0.12 \mathrm{~mm}$

4597 independent reflections

4124 reflections with $I>2 \sigma(I)$

$R_{\text {int }}=0.036$

$\theta_{\text {max }}=30.0^{\circ}, \theta_{\text {min }}=3.0^{\circ}$

$h=-7 \rightarrow 7$

$k=-11 \rightarrow 11$

$l=-25 \rightarrow 25$ 


\section{Refinement}

Refinement on $F^{2}$

Least-squares matrix: full

$R\left[F^{2}>2 \sigma\left(F^{2}\right)\right]=0.030$

$w R\left(F^{2}\right)=0.080$

$S=1.07$

4597 reflections

247 parameters

0 restraints

Primary atom site location: structure-invariant direct methods
Secondary atom site location: difference Fourier map

Hydrogen site location: mixed

$\mathrm{H}$ atoms treated by a mixture of independent and constrained refinement

$w=1 /\left[\sigma^{2}\left(F_{\mathrm{o}}^{2}\right)+(0.0406 P)^{2}+0.3136 P\right]$ where $P=\left(F_{\mathrm{o}}{ }^{2}+2 F_{\mathrm{c}}{ }^{2}\right) / 3$

$(\Delta / \sigma)_{\max }=0.001$

$\Delta \rho_{\max }=0.75 \mathrm{e} \AA^{-3}$

$\Delta \rho_{\min }=-0.34$ e $\AA^{-3}$

Special details

Geometry. All esds (except the esd in the dihedral angle between two 1.s. planes) are estimated using the full covariance matrix. The cell esds are taken into account individually in the estimation of esds in distances, angles and torsion angles; correlations between esds in cell parameters are only used when they are defined by crystal symmetry. An approximate (isotropic) treatment of cell esds is used for estimating esds involving 1.s. planes.

Fractional atomic coordinates and isotropic or equivalent isotropic displacement parameters $\left(\AA^{2}\right)$

\begin{tabular}{|c|c|c|c|c|}
\hline & $x$ & $y$ & $z$ & $U_{\text {iso }} * / U_{\text {eq }}$ \\
\hline $\mathrm{Cl} 1$ & $0.42856(5)$ & $0.16233(3)$ & $-0.07860(2)$ & $0.01668(7)$ \\
\hline $\mathrm{Cl} 2$ & $1.13911(5)$ & $0.28348(3)$ & $0.52483(2)$ & $0.01730(7)$ \\
\hline O1 & $0.03685(16)$ & $-0.10701(11)$ & $-0.14946(5)$ & $0.02014(18)$ \\
\hline $\mathrm{O} 2$ & $0.33010(15)$ & $0.24516(10)$ & $0.08473(5)$ & $0.01729(17)$ \\
\hline $\mathrm{O} 3$ & $1.55337(16)$ & $0.23416(11)$ & $0.62981(5)$ & $0.01865(17)$ \\
\hline $\mathrm{O} 4$ & $1.14201(15)$ & $0.00027(10)$ & $0.38854(5)$ & $0.01586(16)$ \\
\hline $\mathrm{O} 5$ & $0.18017(16)$ & $0.33437(10)$ & $0.25526(5)$ & $0.01818(17)$ \\
\hline H5 & $0.118(3)$ & $0.263(2)$ & $0.2115(10)$ & $0.027 *$ \\
\hline O6 & $0.74923(17)$ & 0.40347 (11) & $0.05992(5)$ & $0.02044(18)$ \\
\hline H6 & $0.617(3)$ & $0.350(2)$ & $0.0681(11)$ & $0.031 *$ \\
\hline N1 & $0.76255(18)$ & $0.16991(12)$ & $0.35832(6)$ & 0.01695 (19) \\
\hline H1 & $0.896(3)$ & $0.128(2)$ & $0.3779(10)$ & $0.025^{*}$ \\
\hline $\mathrm{N} 2$ & $1.04584(18)$ & $0.71260(12)$ & $0.25721(6)$ & $0.01696(19)$ \\
\hline $\mathrm{H} 2$ & $1.114(3)$ & $0.777(2)$ & $0.2998(10)$ & $0.025^{*}$ \\
\hline $\mathrm{C} 1$ & $0.02618(19)$ & $-0.05358(13)$ & $-0.08002(6)$ & $0.01355(19)$ \\
\hline $\mathrm{C} 2$ & $0.19204(19)$ & $0.07509(13)$ & $-0.03554(6)$ & $0.01332(19)$ \\
\hline $\mathrm{C} 3$ & $0.18242(19)$ & 0.13365 (13) & $0.04220(6)$ & 0.01289 (19) \\
\hline $\mathrm{C} 4$ & $1.52060(19)$ & $0.12916(13)$ & $0.56903(6)$ & 0.01293 (19) \\
\hline $\mathrm{C} 5$ & $1.33437(19)$ & $0.12624(13)$ & $0.51055(6)$ & $0.01346(19)$ \\
\hline C6 & $1.30221(19)$ & $0.00728(13)$ & $0.44252(6)$ & $0.01271(19)$ \\
\hline $\mathrm{C} 7$ & $0.6571(2)$ & $0.30150(14)$ & $0.39505(7)$ & $0.0178(2)$ \\
\hline $\mathrm{H} 7$ & 0.719110 & 0.355184 & 0.445342 & $0.021^{*}$ \\
\hline $\mathrm{C} 8$ & $0.4617(2)$ & 0.35945 (14) & $0.36116(7)$ & $0.0169(2)$ \\
\hline $\mathrm{H} 8$ & 0.390102 & 0.453256 & 0.387366 & $0.020^{*}$ \\
\hline $\mathrm{C} 9$ & $0.3693(2)$ & $0.27801(13)$ & $0.28719(6)$ & $0.0141(2)$ \\
\hline $\mathrm{C} 10$ & $0.4863(2)$ & 0.14337 (14) & $0.24949(6)$ & $0.0169(2)$ \\
\hline $\mathrm{H} 10$ & 0.430463 & 0.088006 & 0.198898 & $0.020^{*}$ \\
\hline $\mathrm{C} 11$ & $0.6819(2)$ & $0.09297(15)$ & $0.28659(7)$ & $0.0180(2)$ \\
\hline
\end{tabular}




$\begin{array}{lllll}\text { H11 } & 0.761693 & 0.002289 & 0.261273 & 0.022^{*} \\ \text { C12 } & 0.8247(2) & 0.62477(14) & 0.25631(6) & 0.0169(2) \\ \text { H12 } & 0.743357 & 0.635815 & 0.301370 & 0.020^{*} \\ \text { C13 } & 0.7170(2) & 0.51997(14) & 0.19096(6) & 0.0152(2) \\ \text { H13 } & 0.560916 & 0.459117 & 0.190415 & 0.018^{*} \\ \text { C14 } & 0.8392(2) & 0.50334(13) & 0.12492(6) & 0.0145(2) \\ \text { C15 } & 1.0695(2) & 0.59750(15) & 0.12741(7) & 0.0178(2) \\ \text { H15 } & 1.155223 & 0.590054 & 0.083294 & 0.021^{*} \\ \text { C16 } & 1.1663(2) & 0.69955(15) & 0.19462(7) & 0.0181(2) \\ \text { H16 } & 1.321913 & 0.762460 & 0.197088 & 0.022^{*}\end{array}$

Atomic displacement parameters $\left(\AA^{2}\right)$

\begin{tabular}{lllllll}
\hline & $U^{11}$ & $U^{22}$ & $U^{33}$ & $U^{12}$ & $U^{13}$ & $U^{23}$ \\
\hline C11 & $0.01602(12)$ & $0.01837(13)$ & $0.01388(12)$ & $-0.00454(9)$ & $0.00310(9)$ & $0.00181(9)$ \\
C12 & $0.01776(13)$ & $0.01555(12)$ & $0.01668(13)$ & $0.00527(9)$ & $-0.00194(9)$ & $-0.00109(9)$ \\
O1 & $0.0204(4)$ & $0.0252(4)$ & $0.0108(4)$ & $-0.0062(3)$ & $0.0022(3)$ & $-0.0016(3)$ \\
O2 & $0.0165(4)$ & $0.0179(4)$ & $0.0136(4)$ & $-0.0054(3)$ & $-0.0007(3)$ & $-0.0014(3)$ \\
O3 & $0.0202(4)$ & $0.0181(4)$ & $0.0140(4)$ & $0.0038(3)$ & $-0.0031(3)$ & $-0.0038(3)$ \\
O4 & $0.0164(4)$ & $0.0164(4)$ & $0.0129(4)$ & $0.0021(3)$ & $-0.0034(3)$ & $0.0005(3)$ \\
O5 & $0.0183(4)$ & $0.0175(4)$ & $0.0162(4)$ & $0.0037(3)$ & $-0.0050(3)$ & $-0.0004(3)$ \\
O6 & $0.0196(4)$ & $0.0230(4)$ & $0.0135(4)$ & $-0.0083(3)$ & $0.0008(3)$ & $-0.0029(3)$ \\
N1 & $0.0157(4)$ & $0.0177(4)$ & $0.0181(5)$ & $0.0022(3)$ & $-0.0003(4)$ & $0.0057(4)$ \\
N2 & $0.0177(4)$ & $0.0173(4)$ & $0.0128(4)$ & $-0.0016(3)$ & $-0.0028(4)$ & $-0.0006(3)$ \\
C1 & $0.0126(4)$ & $0.0151(5)$ & $0.0117(5)$ & $-0.0008(4)$ & $0.0003(4)$ & $0.0014(4)$ \\
C2 & $0.0118(4)$ & $0.0151(4)$ & $0.0121(5)$ & $-0.0025(4)$ & $0.0018(4)$ & $0.0023(4)$ \\
C3 & $0.0120(4)$ & $0.0131(4)$ & $0.0126(5)$ & $-0.0004(3)$ & $0.0000(4)$ & $0.0019(4)$ \\
C4 & $0.0134(4)$ & $0.0129(4)$ & $0.0116(5)$ & $0.0001(4)$ & $0.0011(4)$ & $0.0012(4)$ \\
C5 & $0.0135(4)$ & $0.0128(4)$ & $0.0133(5)$ & $0.0028(4)$ & $-0.0001(4)$ & $0.0007(4)$ \\
C6 & $0.0122(4)$ & $0.0124(4)$ & $0.0126(5)$ & $-0.0005(3)$ & $-0.0001(4)$ & $0.0020(4)$ \\
C7 & $0.0200(5)$ & $0.0153(5)$ & $0.0162(5)$ & $0.0000(4)$ & $-0.0032(4)$ & $0.0016(4)$ \\
C8 & $0.0196(5)$ & $0.0141(5)$ & $0.0154(5)$ & $0.0020(4)$ & $-0.0011(4)$ & $0.0003(4)$ \\
C9 & $0.0144(4)$ & $0.0131(4)$ & $0.0146(5)$ & $0.0000(4)$ & $0.0006(4)$ & $0.0032(4)$ \\
C10 & $0.0201(5)$ & $0.0171(5)$ & $0.0125(5)$ & $0.0027(4)$ & $0.0010(4)$ & $0.0012(4)$ \\
C11 & $0.0197(5)$ & $0.0180(5)$ & $0.0170(5)$ & $0.0045(4)$ & $0.0043(4)$ & $0.0034(4)$ \\
C12 & $0.0170(5)$ & $0.0198(5)$ & $0.0129(5)$ & $0.0010(4)$ & $0.0016(4)$ & $0.0015(4)$ \\
C13 & $0.0132(4)$ & $0.0164(5)$ & $0.0147(5)$ & $-0.0011(4)$ & $0.0008(4)$ & $0.0019(4)$ \\
C14 & $0.0149(5)$ & $0.0137(4)$ & $0.0129(5)$ & $-0.0006(4)$ & $-0.0006(4)$ & $0.0002(4)$ \\
C15 & $0.0156(5)$ & $0.0205(5)$ & $0.0148(5)$ & $-0.0039(4)$ & $0.0023(4)$ & $0.0001(4)$ \\
C16 & $0.0146(5)$ & $0.0191(5)$ & $0.0181(5)$ & $-0.0032(4)$ & $-0.0001(4)$ & $0.0010(4)$ \\
& & & & & & \\
\hline
\end{tabular}

Geometric parameters $\left(\AA,{ }^{\circ}\right)$

\begin{tabular}{llll}
\hline $\mathrm{C} 11-\mathrm{C} 2$ & $1.7363(11)$ & $\mathrm{C} 4-\mathrm{C} 5$ & $1.4165(14)$ \\
$\mathrm{C} 12-\mathrm{C} 5$ & $1.7438(11)$ & $\mathrm{C} 4-\mathrm{C} 6$ & $1.5426(15)$ \\
$\mathrm{O} 1-\mathrm{C} 1$ & $1.2508(13)$ & $\mathrm{C} 5-\mathrm{C} 6$ & $1.3924(15)$ \\
$\mathrm{O} 2-\mathrm{C} 3$ & $1.2532(12)$ & $\mathrm{C} 7-\mathrm{C} 8$ & $1.3722(16)$ \\
$\mathrm{O} 3-\mathrm{C} 4$ & $1.2390(13)$ & $\mathrm{C} 7-\mathrm{H} 7$ & 0.9500
\end{tabular}




\begin{tabular}{|c|c|c|c|}
\hline $\mathrm{O} 4-\mathrm{C} 6$ & $1.2586(13)$ & $\mathrm{C} 8-\mathrm{C} 9$ & $1.4035(15)$ \\
\hline $\mathrm{O} 5-\mathrm{C} 9$ & $1.3223(13)$ & $\mathrm{C} 8-\mathrm{H} 8$ & 0.9500 \\
\hline $\mathrm{O} 5-\mathrm{H} 5$ & $0.907(18)$ & $\mathrm{C} 9-\mathrm{C} 10$ & $1.4065(16)$ \\
\hline $\mathrm{O} 6-\mathrm{C} 14$ & $1.3208(13)$ & $\mathrm{C} 10-\mathrm{C} 11$ & $1.3703(16)$ \\
\hline $\mathrm{O} 6-\mathrm{H} 6$ & $0.852(19)$ & $\mathrm{C} 10-\mathrm{H} 10$ & 0.9500 \\
\hline $\mathrm{N} 1-\mathrm{C} 11$ & $1.3456(15)$ & $\mathrm{C} 11-\mathrm{H} 11$ & 0.9500 \\
\hline $\mathrm{N} 1-\mathrm{C} 7$ & $1.3469(15)$ & $\mathrm{C} 12-\mathrm{C} 13$ & $1.3693(15)$ \\
\hline $\mathrm{N} 1-\mathrm{H} 1$ & $0.918(18)$ & $\mathrm{C} 12-\mathrm{H} 12$ & 0.9500 \\
\hline $\mathrm{N} 2-\mathrm{C} 16$ & $1.3430(16)$ & $\mathrm{C} 13-\mathrm{C} 14$ & 1.4009 (16) \\
\hline $\mathrm{N} 2-\mathrm{C} 12$ & $1.3511(15)$ & $\mathrm{C} 13-\mathrm{H} 13$ & 0.9500 \\
\hline $\mathrm{N} 2-\mathrm{H} 2$ & $0.877(18)$ & $\mathrm{C} 14-\mathrm{C} 15$ & $1.4126(15)$ \\
\hline $\mathrm{C} 1-\mathrm{C} 2$ & $1.3997(14)$ & $\mathrm{C} 15-\mathrm{C} 16$ & $1.3671(15)$ \\
\hline $\mathrm{C} 1-\mathrm{C} 3^{\mathrm{i}}$ & $1.5410(15)$ & $\mathrm{C} 15-\mathrm{H} 15$ & 0.9500 \\
\hline $\mathrm{C} 2-\mathrm{C} 3$ & $1.3970(15)$ & $\mathrm{C} 16-\mathrm{H} 16$ & 0.9500 \\
\hline $\mathrm{C} 9-\mathrm{O} 5-\mathrm{H} 5$ & $111.3(11)$ & $\mathrm{C} 8-\mathrm{C} 7-\mathrm{H} 7$ & 119.4 \\
\hline $\mathrm{C} 14-\mathrm{O} 6-\mathrm{H} 6$ & $107.1(13)$ & $\mathrm{C} 7-\mathrm{C} 8-\mathrm{C} 9$ & 118.99 (11) \\
\hline $\mathrm{C} 11-\mathrm{N} 1-\mathrm{C} 7$ & $120.85(10)$ & $\mathrm{C} 7-\mathrm{C} 8-\mathrm{H} 8$ & 120.5 \\
\hline $\mathrm{C} 11-\mathrm{N} 1-\mathrm{H} 1$ & $114.5(11)$ & $\mathrm{C} 9-\mathrm{C} 8-\mathrm{H} 8$ & 120.5 \\
\hline $\mathrm{C} 7-\mathrm{N} 1-\mathrm{H} 1$ & $124.6(11)$ & $\mathrm{O} 5-\mathrm{C} 9-\mathrm{C} 8$ & $118.46(10)$ \\
\hline $\mathrm{C} 16-\mathrm{N} 2-\mathrm{C} 12$ & $121.30(10)$ & $\mathrm{O} 5-\mathrm{C} 9-\mathrm{C} 10$ & $122.89(10)$ \\
\hline $\mathrm{C} 16-\mathrm{N} 2-\mathrm{H} 2$ & $119.3(12)$ & $\mathrm{C} 8-\mathrm{C} 9-\mathrm{C} 10$ & $118.62(10)$ \\
\hline $\mathrm{C} 12-\mathrm{N} 2-\mathrm{H} 2$ & $119.4(12)$ & $\mathrm{C} 11-\mathrm{C} 10-\mathrm{C} 9$ & $119.23(10)$ \\
\hline $\mathrm{O} 1-\mathrm{C} 1-\mathrm{C} 2$ & $123.43(10)$ & $\mathrm{C} 11-\mathrm{C} 10-\mathrm{H} 10$ & 120.4 \\
\hline $\mathrm{O} 1-\mathrm{C} 1-\mathrm{C} 3^{\mathrm{i}}$ & $117.79(9)$ & $\mathrm{C} 9-\mathrm{C} 10-\mathrm{H} 10$ & 120.4 \\
\hline $\mathrm{C} 2-\mathrm{C} 1-\mathrm{C} 3^{\mathrm{i}}$ & $118.79(9)$ & $\mathrm{N} 1-\mathrm{C} 11-\mathrm{C} 10$ & $121.03(11)$ \\
\hline $\mathrm{C} 3-\mathrm{C} 2-\mathrm{C} 1$ & $123.56(10)$ & $\mathrm{N} 1-\mathrm{C} 11-\mathrm{H} 11$ & 119.5 \\
\hline $\mathrm{C} 3-\mathrm{C} 2-\mathrm{C} 11$ & $118.10(8)$ & $\mathrm{C} 10-\mathrm{C} 11-\mathrm{H} 11$ & 119.5 \\
\hline $\mathrm{C} 1-\mathrm{C} 2-\mathrm{C} 11$ & $118.31(8)$ & $\mathrm{N} 2-\mathrm{C} 12-\mathrm{C} 13$ & $120.52(11)$ \\
\hline $\mathrm{O} 2-\mathrm{C} 3-\mathrm{C} 2$ & $125.93(10)$ & $\mathrm{N} 2-\mathrm{C} 12-\mathrm{H} 12$ & 119.7 \\
\hline $\mathrm{O} 2-\mathrm{C} 3-\mathrm{C}^{\mathrm{i}}$ & $116.43(9)$ & $\mathrm{C} 13-\mathrm{C} 12-\mathrm{H} 12$ & 119.7 \\
\hline $\mathrm{C} 2-\mathrm{C} 3-\mathrm{C} 1^{\mathrm{i}}$ & $117.64(9)$ & $\mathrm{C} 12-\mathrm{C} 13-\mathrm{C} 14$ & $119.37(10)$ \\
\hline $\mathrm{O} 3-\mathrm{C} 4-\mathrm{C} 5$ & $124.83(10)$ & $\mathrm{C} 12-\mathrm{C} 13-\mathrm{H} 13$ & 120.3 \\
\hline $\mathrm{O} 3-\mathrm{C} 4-\mathrm{C}^{\mathrm{ii}}$ & $116.58(9)$ & $\mathrm{C} 14-\mathrm{C} 13-\mathrm{H} 13$ & 120.3 \\
\hline $\mathrm{C} 5-\mathrm{C} 4-\mathrm{C} 6^{\mathrm{ii}}$ & $118.59(9)$ & $\mathrm{O} 6-\mathrm{C} 14-\mathrm{C} 13$ & $123.08(10)$ \\
\hline $\mathrm{C} 6-\mathrm{C} 5-\mathrm{C} 4$ & $123.72(10)$ & $\mathrm{O} 6-\mathrm{C} 14-\mathrm{C} 15$ & $118.05(10)$ \\
\hline $\mathrm{C} 6-\mathrm{C} 5-\mathrm{Cl} 2$ & $118.75(8)$ & $\mathrm{C} 13-\mathrm{C} 14-\mathrm{C} 15$ & $118.87(10)$ \\
\hline $\mathrm{C} 4-\mathrm{C} 5-\mathrm{Cl} 2$ & $117.52(8)$ & $\mathrm{C} 16-\mathrm{C} 15-\mathrm{C} 14$ & $118.63(11)$ \\
\hline $\mathrm{O} 4-\mathrm{C} 6-\mathrm{C} 5$ & $126.22(10)$ & $\mathrm{C} 16-\mathrm{C} 15-\mathrm{H} 15$ & 120.7 \\
\hline $\mathrm{O} 4-\mathrm{C} 6-\mathrm{C}^{\mathrm{ii}}$ & $116.09(9)$ & $\mathrm{C} 14-\mathrm{C} 15-\mathrm{H} 15$ & 120.7 \\
\hline $\mathrm{C} 5-\mathrm{C} 6-\mathrm{C}^{\mathrm{ii}}$ & $117.69(9)$ & $\mathrm{N} 2-\mathrm{C} 16-\mathrm{C} 15$ & $121.29(10)$ \\
\hline $\mathrm{N} 1-\mathrm{C} 7-\mathrm{C} 8$ & $121.23(11)$ & $\mathrm{N} 2-\mathrm{C} 16-\mathrm{H} 16$ & 119.4 \\
\hline $\mathrm{N} 1-\mathrm{C} 7-\mathrm{H} 7$ & 119.4 & $\mathrm{C} 15-\mathrm{C} 16-\mathrm{H} 16$ & 119.4 \\
\hline $\mathrm{O} 1-\mathrm{C} 1-\mathrm{C} 2-\mathrm{C} 3$ & $-179.19(11)$ & $\mathrm{C} 11-\mathrm{N} 1-\mathrm{C} 7-\mathrm{C} 8$ & $1.17(18)$ \\
\hline $\mathrm{C} 3-\mathrm{C} 1-\mathrm{C} 2-\mathrm{C} 3$ & $1.29(18)$ & $\mathrm{N} 1-\mathrm{C} 7-\mathrm{C} 8-\mathrm{C} 9$ & $0.81(18)$ \\
\hline $\mathrm{O} 1-\mathrm{C} 1-\mathrm{C} 2-\mathrm{C} 11$ & $-1.30(16)$ & $\mathrm{C} 7-\mathrm{C} 8-\mathrm{C} 9-\mathrm{O} 5$ & $179.64(10)$ \\
\hline $\mathrm{C} 33^{\mathrm{i}}-\mathrm{C} 1-\mathrm{C} 2-\mathrm{C} 11$ & $179.17(7)$ & $\mathrm{C} 7-\mathrm{C} 8-\mathrm{C} 9-\mathrm{C} 10$ & $-2.23(17)$ \\
\hline
\end{tabular}




\begin{tabular}{|c|c|c|c|}
\hline $\mathrm{C} 1-\mathrm{C} 2-\mathrm{C} 3-\mathrm{O} 2$ & $178.00(11)$ & $\mathrm{O} 5-\mathrm{C} 9-\mathrm{C} 10-\mathrm{C} 11$ & $179.80(10)$ \\
\hline $\mathrm{C} 11-\mathrm{C} 2-\mathrm{C} 3-\mathrm{O} 2$ & $0.11(16)$ & $\mathrm{C} 8-\mathrm{C} 9-\mathrm{C} 10-\mathrm{C} 11$ & $1.75(17)$ \\
\hline $\mathrm{C} 1-\mathrm{C} 2-\mathrm{C} 3-\mathrm{C}^{\mathrm{i}}$ & $-1.27(17)$ & $\mathrm{C} 7-\mathrm{N} 1-\mathrm{C} 11-\mathrm{C} 10$ & $-1.68(17)$ \\
\hline $\mathrm{C} 11-\mathrm{C} 2-\mathrm{C} 3-\mathrm{C}^{\mathrm{i}}$ & $-179.16(7)$ & $\mathrm{C} 9-\mathrm{C} 10-\mathrm{C} 11-\mathrm{N} 1$ & $0.18(17)$ \\
\hline $\mathrm{O} 3-\mathrm{C} 4-\mathrm{C} 5-\mathrm{C} 6$ & $179.64(11)$ & $\mathrm{C} 16-\mathrm{N} 2-\mathrm{C} 12-\mathrm{C} 13$ & $-0.23(17)$ \\
\hline $\mathrm{C} 6{ }^{\mathrm{ii}}-\mathrm{C} 4-\mathrm{C} 5-\mathrm{C} 6$ & $-0.41(17)$ & $\mathrm{N} 2-\mathrm{C} 12-\mathrm{C} 13-\mathrm{C} 14$ & $0.54(17)$ \\
\hline $\mathrm{O} 3-\mathrm{C} 4-\mathrm{C} 5-\mathrm{Cl} 2$ & $0.75(16)$ & $\mathrm{C} 12-\mathrm{C} 13-\mathrm{C} 14-\mathrm{O} 6$ & $179.17(11)$ \\
\hline $\mathrm{C} 6{ }^{\mathrm{ii}}-\mathrm{C} 4-\mathrm{C} 5-\mathrm{Cl} 2$ & $-179.29(7)$ & $\mathrm{C} 12-\mathrm{C} 13-\mathrm{C} 14-\mathrm{C} 15$ & $-0.92(17)$ \\
\hline $\mathrm{C} 4-\mathrm{C} 5-\mathrm{C} 6-\mathrm{O} 4$ & $-179.11(10)$ & $\mathrm{O} 6-\mathrm{C} 14-\mathrm{C} 15-\mathrm{C} 16$ & $-179.08(11)$ \\
\hline $\mathrm{Cl} 2-\mathrm{C} 5-\mathrm{C} 6-\mathrm{O} 4$ & $-0.23(16)$ & $\mathrm{C} 13-\mathrm{C} 14-\mathrm{C} 15-\mathrm{C} 16$ & $1.01(17)$ \\
\hline $\mathrm{C} 4-\mathrm{C} 5-\mathrm{C} 6-\mathrm{C}^{\mathrm{ii}}$ & $0.40(17)$ & $\mathrm{C} 12-\mathrm{N} 2-\mathrm{C} 16-\mathrm{C} 15$ & $0.33(18)$ \\
\hline $\mathrm{Cl} 2-\mathrm{C} 5-\mathrm{C} 6-\mathrm{C}^{\mathrm{ii}}$ & $179.28(7)$ & $\mathrm{C} 14-\mathrm{C} 15-\mathrm{C} 16-\mathrm{N} 2$ & $-0.72(18)$ \\
\hline
\end{tabular}

Symmetry codes: (i) $-x,-y,-z$; (ii) $-x+3,-y,-z+1$.

Hydrogen-bond geometry $\left(\AA,{ }^{\circ}\right)$

\begin{tabular}{lllll}
\hline$D-\mathrm{H} \cdots A$ & $D-\mathrm{H}$ & $\mathrm{H} \cdots A$ & $D \cdots A$ & $D-\mathrm{H} \cdots A$ \\
\hline $\mathrm{O} 5-\mathrm{H} 5 \cdots \mathrm{O} 1^{\mathrm{i}}$ & $0.905(17)$ & $1.640(17)$ & $2.5208(13)$ & $163.2(17)$ \\
$\mathrm{O} 6-\mathrm{H} 6 \cdots \mathrm{O} 2$ & $0.852(17)$ & $1.800(17)$ & $2.6510(13)$ & $177.3(18)$ \\
$\mathrm{N} 1-\mathrm{H} 1 \cdots \mathrm{O} 4$ & $0.919(17)$ & $1.810(17)$ & $2.7000(13)$ & $162.3(16)$ \\
$\mathrm{N} 2-\mathrm{H} 2 \cdots \mathrm{O} 4$ iii & $0.876(18)$ & $2.156(17)$ & $2.9603(14)$ & $152.3(15)$ \\
$\mathrm{N} 2-\mathrm{H} 2 \cdots \mathrm{O} 3^{\text {iv }}$ & $0.876(18)$ & $2.176(17)$ & $2.8384(14)$ & $132.1(14)$ \\
$\mathrm{C} 7-\mathrm{H} 7 \cdots \mathrm{Cl} 2$ & 0.95 & 2.81 & $3.4540(12)$ & 126 \\
$\mathrm{C} 12-\mathrm{H} 12 \cdots \mathrm{O} 3^{v}$ & 0.95 & 2.32 & $3.1541(14)$ & 146 \\
$\mathrm{C} 13-\mathrm{H} 13 \cdots \mathrm{O} 2$ & 0.95 & 2.49 & $3.1685(14)$ & 128 \\
$\mathrm{C} 16-\mathrm{H} 16 \cdots \mathrm{Cl} 1^{\text {vi }}$ & 0.95 & 2.77 & $3.4427(12)$ & 128
\end{tabular}

Symmetry codes: (i) $-x,-y,-z$; (iii) $x, y+1, z$; (iv) $-x+3,-y+1,-z+1$; (v) $-x+2,-y+1,-z+1$; (vi) $-x+2,-y+1,-z$. 